\title{
LE SILENCE DES LAMBEAUX : LE CADAVRE, LE SÉPULCRE ET LA PHILOSOPHIE DU DROIT
}

\author{
DOI:10.24193/SUBBiur.62(2017).4.1 \\ Published Online: 2017-12-20 \\ Published Print: 2017-12-30
}

Bjarne MELKEVIK*

\begin{abstract}
Over my dead body": the corpse, the sepulchre and the philosophy of law. The author submits "the corpse" to an iusphilosophical reflection by emphasizing how our attitude in this area reveals our humanity and our process of humanization, as well as our psychological and cultural attitudes. First, the author opens a reflection on the corpse in an archaeological and historical-as well as - anthropological and cultural context. Afterwards, he successively examines the corpse in the sepulchre, as a "taboo" object and finally as "desire" rising from ourselves or from others. The author places, literally, the corpse in its place before the law without ever examining positive law. The aim of the article is to make the reflection on the law towards its intimacy as to our conscience of humanity.
\end{abstract}

Keywords: Legal Humanism; Legal Philosophy; Theory of Law; Human remains (mortal remains, cadaver, corpse.) and Law; sepulchre (GB) / sepulcher (US) and Law; Crimes against (indignity to a) corpse; Civil Law.

Résumé : L'auteur soumet "le cadavre " à une réflexion iusphilosophique en insistant sur comment notre attitude dans ce domaine révèle notre humanité et notre processus d'humanisation, de même que nos attitudes psychologiques et culturelles. Dans un premier temps, l'auteur ouvre la réflexion sur le cadavre dans le contexte archéologique et historique de même qu'anthropologique et culturel. Ensuite, il examine successivement le cadavre dans le sépulcre en tant qu'objet " tabou " et enfin en tant que "désir » par rapport à un soi ou à autrui. L'auteur place littéralement le cadavre dans sa place devant le droit sans jamais pour autant examiner le droit positif. L'objectif de l'article c'est de faire un voyage dans la réflexion sur le droit le cheminant vers son intimité avec notre conscience de l'humanité.

Mots clefs : Humanisme juridique ; Philosophie du droit ; Théorie du droit ; cadavre et droit ; sépulcre et droit ; crimes contre un cadavre; droit civil. 
L'objet de notre essai est le cadavre. Celui qui prononce aujourd'hui le mot " cadavre » reconnaît volontiers que celui-ci dispose de deux "vies ", l'une est liée à la nature tandis que l'autre se rattache à la notion de culture. On pourrait également en situer une autre entre les deux susmentionnées. Quant au droit, nous le laissons intentionnellement dans l'antichambre.

Tous les voyageurs des pays lointains ne seront pas de notre bord, mais nous avons toujours eu le sentiment (et l'intuition) qu'en ce qui concerne un pays, une ville ou une contrée humaine, rien ne se dérobe tout à fait ou ne se découvre réellement, qu'après la visite des cimetières.

Si les Grecs de l'Antiquité faisaient la distinction entre les barbares et les gens civilisés, en jugeant les coutumes de sépultures, l'honneur rendu aux morts, l'idée de voir la question de culture, de civilisation, s'exposant dans la pitié et la sérénité que témoignent (ou non) le respect accordé aux morts, aux cadavres, n'apparaît, de ce fait, pas si étonnant. Après tout, la culture et la civilisation ne se reflètent-elles pas mieux, et après réflexion, plus honnêtement, dans le respect que les vivants rendent à celui qui est mort, à son corps, à son cadavre ? Quand les splendeurs touristiques officielles, pour ne pas dire les tape-à-l'œil (à la mode du sacrifice " rendu à La Patrie »), ne respirent le plus souvent que de la malhonnêteté et de l'orgueil, existe-t-il quelque chose de plus personnel et intime que les honneurs que les fils et les filles ont rendus et rendent depuis des temps immémoriaux à leurs défunts?

À notre tour d'observer, de contempler et surtout de réfléchir, à notre attitude à l'égard du cadavre et de ce que nous accomplissons avec (et pour) le cadavre humain. Tertullien nous le rappelle : le corps humain en putréfaction, le cadavre après la maladie, l'accident, le meurtre ou l'épuisement du fardeau des jours et des durs labeurs « retourne à la terre, son premier élément, pour prendre le nom de cadavre; même ce nom de cadavre ne lui demeurera pas longtemps: il deviendra un je ne sais quoi qui n'a plus de nom dans aucune langue ${ }^{1}$. Et avec ces mots si justes se résume l'égalité parfaite, la vraie justice, qu'attend au bout du chemin tout être humain. Comme tout individu honnête et intelligent le sait, c'est uniquement notre commune mortalité qui nous authentifie grâce à la Grande Faucheuse. Nous devenons des égaux. Hors la mort, hors l'égalité !

Bien évidemment, tout cadavre n'a pas forcément droit ni à l'honneur d'un dernier adieu post-mortem des vivants ni une sépulture. En ce sens, n'est-il pas significatif d'observer comment plusieurs personnes témoignent d'un blocage psychologique et d'une impossibilité spirituelle (et de piété religieuse) qui les empêchent de faire leur deuil sans la présence d'un corps mort, un corps à enterrer, un cadavre ? Celui qui a perdu un être cher, ses parents, ou pire son enfant ${ }^{2}$ sait au fond de lui-même que le "cadavre " n'est pas uniquement une "chose » bonne pour retourner à la terre. II s'agit d'une partie de son histoire, de sa mémoire et même de son existence, dans la façon de se comprendre soimême et de concevoir les autres. Que l'État ou, plus adéquatement, la communauté des cosociétaires, s'en mêle en introduisant des obligations hygiéniques et sanitaires, n'obscurcit en rien l'interrogation de savoir " que faire du cadavre », le " corps du défunt " ? 
On touche ici à l'intimité de l'âme et ceci nous force à réfléchir et à nous interroger, à la limite de notre désespoir, sur la vie et sur la mort. La question du cadavre, du corps postmortem, n'est, de toute évidence, pas contingente à une question pratico technique (et de thanatologie $\mathrm{e}^{3}$ ) ou encore sanitaire et hygiénique. Au contraire, elle relève, d'une façon plus pertinente, de la culture, de la civilisation et de la représentation de soi-même à la lignée générationnelle (parentale) et, notamment, au respect des aïeuls ou encore de la " nation » (en rappelant que le sens de ce mot s'enracine dans le mot latin nascěre que l'on peut attribuer au verbe naître, au mot origine, et également au " provenir » de ceux qui sont nés dans ladite " nation »). Lorsque l'on se demande " que faire avec le cadavre ? " se soulève, de ce fait, une interrogation qui rejoint instantanément l'intimité de l'âme, la profondeur de notre conscience à tous et qui questionne notre humanité.

C'est sur ce fond qu'interviennent nos réflexions, des pensées fatalement circonscrites et brèves (et surtout reprochables, coupables, à l'égard de tout ce que nous n'examinerons pas, de tout ce que nous écartons et de tout ce qui pouvait être mentionné). Nous tenterons, en effet, par la suite, de préparer le terrain et d'ouvrir la porte, pour la pensée juridique (et iusphilosophique), en ce qui concerne «le droit et le cadavre ».

En focalisant, comme nous en avons l'intention, nos attitudes, nos mentalités, notre " pitié » à l'égard du corps post-mortem, du cadavre, notre essai n'intervient qu'en amont, en tant que réflexion culturelle et civilisatrice. II adopte un itinéraire exploratoire où il s'agit de réfléchir, d'abord, sur le cadavre dans sa sépulture ; ensuite de s'intéresser aux " interdits ", aux tabous, touchant le corps mort, le cadavre; et, enfin, d'examiner le désir (chez soi ou chez les autres) d'échapper au destin fatal de putréfaction et au retour à la terre. Au préalable, quelques précisions d'ordre historique et anthropologique concernant la gérance des " restes ", du " cadavre ", s'imposent, afin de mieux comprendre le sens de nos propos à venir.

\section{D'être anthropoïde et d'affirmation d'humanité}

Celui qui se préoccupe d'une question si délicate, si sensible et, surtout, si humaine, que la destinée du cadavre humain et de nos attitudes et de nos pratiques à son égard, risque fort de se décourager. II y a tant de choses à dire, à explorer et à examiner, et cela autant au niveau historique qu'anthropologique! Les pratiques, les attitudes et les mentalités, tant humaines, anthropologiques, géographiques, que culturelles, coutumières, spirituelles ou encore religieuses, varient historiquement presque à l'infini et chaque élément nécessite souvent des explications très complexes et très approfondies pour arriver à en déchiffrer leurs sens (supposément) véritables.

D'un côté, nous avons les faits, tandis que de l'autre, nous avons les pratiques culturelles, ethnographiques, religieuses, symboliques, etc. Ces deux côtés font l'objet d'interprétations et de constructions tenant à la compréhension bien diversifiées (sinon controversées) et différentes. Or, dès que les faits (et les objets réels) sont réalisés, fabriqués, produits, ils deviennent eux-mêmes des artefacts culturels, ethnographiques, religieux, symboliques. En ce sens, pour être plus clair, le vrai est ce qui est fait (la « verum 
factum $»^{4}$ ) et le "ce qui est fait », est ce que leurs producteurs ont réalisé en tant que " fait » pour eux et à partir de leurs prémisses. II s'en suit qu'il n'y a pas de " connaissance en soi " (et aucune théorie de la connaissance ou connaissance théorique n'est possible non plus ; ce que n'ont pas compris nos constructivistes modernes) et donc que toute connaissance doit être " pratique » et se concrétiser sous la forme d'une interrogation de savoir comment le sujet et les individus en ont fait l'objet.

Spécifiquement, en ce qui concerne les cadavres, les faits s'accompagnent de pratiques culturelles, ethnographiques, religieuses, symboliques, etc. très diversifiées et pouvant faire l'objet d'interprétations très divergentes. Exemplifions-le avec la momification en Égypte pharaonique ${ }^{5}$ qui a subi des changements significatifs au niveau technique, religieux et symbolique pendant plus que quatre mille ans (jusqu'à, grosso modo, l'invasion islamiste au $7^{\mathrm{e}}$ siècle après J.-C.). La momification pharaonique était, de toute évidence, beaucoup plus qu'une technique (utilisant des matériaux rares et coûteux) et impliquait surtout une variété complexe de croyances religieuses qui s'accompagnaient de coutumes funéraires et de dispositions d'embaumement ritualisées très sophistiquée ${ }^{6}$. Mentionnons, dans le même temps, la Chine antique, la Grèce antique, la Rome antique et d'autres anciennes cultures où le respect du corps du défunt a été la pierre angulaire de la " vénération des ancêtres » et servait le rôle d'une religion où se conjuguaient les mythes, les philosophies et les légendes.

Deux aspects méritent maintenant une attention particulière, d'abord la diversité culturelle et coutumière de cette humanisation, vue à partir des thanatopraxies et, ensuite, l'humanisation en tant que miroir d'attitudes, de conscience, de conduites humaines diversifiées à l'égard du cadavre. Les deux aspects se confondent nécessairement et se comprennent, formant le côté dextre et senestre de la même chose.

\section{L'histoire et le cadavre}

Il y a, nous l'avons déjà indiqué, quelque chose d'intrinsèquement " humain " qui se révèle dans notre attitude à l'égard du corps post-mortuaire, à l'égard du cadavre. L'éminent philosophe italien Giambattista Vico (1668 - 1744) considérait que le sépulcre (et le rituel implicite qui l'accompagne) révèle la prise de conscience nécessaire (de soi et de sa propre mortalité) conduisant à l'humanisation de l'humain et également à l'inauguration d'un processus d'apprentissage de civilisation, de culture, de mœurs et de respect ${ }^{7}$. Nous le suivons volontairement et il en découle que ce n'est ni le langage, ni la logique, ni l'homo faber (c.-àd. I'homme comme faiseur d'outils), ni non plus notre intelligence affirmée "abstraite " qui distingue rationnellement l'homme de la bête, sinon l'humain prenant conscience de la mort des siens et qui arrive à concevoir la nécessité de créer un éloignement symbolique et culturel entre les vivants et le corps mort. De ce fait, l'humanoïde se distingue et se sépare de plus en plus, par des étapes emblématiques, de la bête, de l'animal qu'il a été et il devient proprement humain en s'établissant et en s'éduquant devant une conscience d'éloignement symbolique qui est représentée par un acte délibéré de séparation consciente entre le cadavre et le vivant. 
Vico a vu juste ! Par l'image de l'humain qui accepte de prendre en charge consciencieusement le corps-mort, le cadavre, c'est effectivement la logique de la séparation, de l'éloignement, de la distanciation, de la délocalisation, de la création d'un espace culturel et d'une temporalité symbolique séparant les morts des vivants, et les différentes pratiques de traitement du cadavre, qui prend tous leurs sens. $\mathrm{Si}$, en effet, aucune donnée historique ou anthropologique ne nous permet avec certitude de prétendre (et cela de loin) à une pratique unique, l'insistance sur "une prise de conscience " (nécessairement très diversifiée, car " humaine ") qui, de facto, sert à séparer les vivants des morts, se constate dans un nombre impressionnant de pratiques bien différentes autour du monde. C'est une variété de thanatopraxies presque à l'infinie, que nous rencontrons au cours de l'histoire et autour du monde, et cela depuis l'aube de l'humanité. Mais toujours, soulignons-le, en établissant des symboliques et des cultures autour de ces thanatopraxies.

Retenons dans la perspective de l'anthropologie "des peuples sans écriture ", en paraphrasant Claude Lévi-Strauss ${ }^{8}$, ou encore des peuplades "sauvages " (ainsi nommées par les philosophes des Lumières, et "renommées " par leurs successeurs !) ayant été découverts et étudiés dans le temps historique récent, qu'il est possible de formuler, nous semble-t-il, la thèse que l'éloignement se pratique d'abord de manière assez concrète et matérielle : la tribu laissait le corps-mort là où il tombait pour continuer son trek ou si elle constatait que le vieillard ou le malade n'arriverait plus à les suivre, il était laissé là pour qu'il meure sur place ${ }^{9}$. On peut prendre pour exemple la tribu amérindienne (en Amazonie) qui quittait le village où une personne y décédait pour s'installer ailleurs en laissant, de ce fait, au cadavre l'ancien village où une putréfaction commune se chargerait de tout faire disparaître naturellement et physiquement. La manutention, le transport, d'un corps mort (et le toilettage funéraire) vers un endroit réservé aux morts (c.-à-d. grottes, puits, montagne, etc.) - souvent désigné ultérieurement comme tabou ou sacré - se caractérise plutôt comme un progrès d'humanisation et le signe de l'impossibilité pratique de quitter et d'abandonner un village qui est devenu un lieu nécessaire pour la survie alimentaire et la sécurité de l'habitat.

Anthropologiquement, cela s'accompagnait surtout de thanatopraxies ${ }^{10}$ instaurant le retour d'un cadavre vers la nature animale. Ces formes de pratiques zoothanalogiques consistaient à un retour " en chair » à la nature et se faisaient en donnant et en offrant réellement le corps du mort en pâturage, en nourriture, aux animaux, aux oiseaux ou encore aux poissons. C'était d'ailleurs, jusqu'à présent, la tradition maritime en ce qui concerne les morts en haute mer - autant dans la marine militaire que sur les bateaux civils de transport. S'ajoutent ici des mythes, des légendes, des narrations mémorielles, des " peuples ichtyophages ${ }^{11}$ qui conviaient, semble-t-il, les poissons à se repaître du corps de leurs défunts. Mais comment distinguer le réel de l'imaginaire quand nous n'avons que des mythes et des légendes?

Plus solide et factuelle se révèle la pratique zoothanalogique consistant à offrir le cadavre aux animaux. C'était d'ailleurs, jusqu'au $19^{\mathrm{e}}$ siècle, la pratique mortuaire coutumière des Amérindiens des plaines en Amérique du Nord. Ces peuples nomades exposaient le corps mort sur des claies surélevées et les cadavres servaient de pâturage pour le plaisir des oiseaux. Mentionnons également les adeptes de Zarathoustra (les mazdéens et les parsis) ${ }^{12}$ 
qui exposaient, hier comme aujourd'hui, les cadavres dans des Tours de silence (ou « de mort ») pour servir de nourriture aux vautours. Les Tours de silence servent, en pratique, à ce que l'on pourrait qualifier de " plateau de chair » où, lorsque tout a été mangé, il ne s'agit plus que de précipiter au fond d'un puits les os immangeables ${ }^{13}$. Aujourd'hui, cette pratique s'accomplit naturellement tous les jours chez les mazdéens en Inde, notamment à Bombay (Mumbai), et à l'indifférence (presque) générale des citoyens de la ville ${ }^{14}$. Or, les " tours de silence " semblent être, pour les adeptes zoroastriens, une métamorphose, une pratique zoothanalogique plus ancienne, consistant à jeter le cadavre aux animaux des alentours. L'ancienne pratique des zoroastriens / mazdéens consistait en effet, comme cela se dit, à " jeter le cadavre en pâture » aux chiens (errants) - ce qui était effectivement l'autre versant de leur zoothanatologie - et qui a été repris proverbialement ${ }^{15}$, dans un cadre non zoroastrien, par l'expression populaire : jeter le cadavre aux chiens ${ }^{16}$.

La zoothanatologie est mal considérée aujourd'hui et semble circonscrite culturellement à la pratique historique des Perses (Iraniens) et la religion zoroastrienne / mazdéenne contemporaine. Si cette forme d'éloignement du cadavre confirme certainement la thèse de Giambattista Vico susmentionnée, force est toutefois de croire que ce jugement négatif engage aujourd'hui l'individu dans un plus grand respect aux cadavres des siens et à un niveau de distanciation élargie.

Reste à savoir si les premiers cadavres découverts enterrés sont ensevelis par une intention humaine ou par accident ou incident naturel. Peut-être ne s'agit-il que d'un cadavre délaissé ou d'un individu que la nature a enterrés: un accident dans un marécage, une avalanche de neige ou de boue, un accident de chasse, une chute fatale, une mort dans une grotte ou une " cavité ", etc. ? Les archéologues de la préhistoire ne se s'aventurent guère, en règle générale, au-dessus de 100.000 ou 80.000 av. J.-C., dans la confirmation d'une volonté (ou d'un acte délibéré) humaine claire et intentionnée d'enterrer un défunt ${ }^{17}$. Conscients du fait qu'un cadavre ne se conserve que difficilement ou simplement pas du tout, sauf sous des conditions extrêmement exceptionnelles (et à ce moment-là, souvent sous forme de " fossilisation " de certains fragments ossuaires), une telle logique de datation est de première importance. Elle nous confirme que c'est, avant tout, la volonté d'éloignement du corps humain mort des prédateurs qui pousse l'humain vers l'enterrement, sinon vers l'incinération.

En étudiant les pratiques de l'enterrement d'un cadavre, c'est une préhistoire fabuleuse, magnifique et intrigante de l'homme qui s'offre à nos yeux et à nos pensées.

La Préhistoire (3000 ans avant J. C.) c'est comme un puzzle où nous ne savons pas combien de pièces existent, ni où elles se trouvent, ni sous quelle forme et, en règle générale, où nous n'avons qu'une idée souvent fragmentaire et brouillée de l'ensemble. Il convient, en ce sens, de ne jamais perdre de vue, au risque de se rendre coupable d'un crime de lèsemajesté historique, que c'est souvent par la découverte, la fouille, l'archéologie, des stèles, des cryptes, des tumulis, des cairns, etc., et des tombeaux où le cadavre a été réduit plus ou moins en poussière, que nous connaissons certains aspects de la préhistoire (de même que de l'histoire) humaine. Des pans entiers de cette préhistoire n'ont pu être étudiés et compris que partiellement, par la découverte de sépulcres et par leurs emplacements, leurs techniques de construction et les mots ou les signes qui s'y trouvaient. 
Des civilisations entières n'ont pu être connues et situées géographiquement et culturellement que par des sépulcres retrouvés. Et la raison se situe dans le fait que l'enterrement du corps défunt se fait depuis fort longtemps et on l'accompagnait d'artefacts (vêtements, armes, ustensiles, etc.) souvent destinés à servir le mort dans son " autre " vie. C'est ce qui permet une identification et une " historisation » d'un peuple, d'une culture.

Cette dernière idée mentionnée, la croyance d'une « vie » post-mortem possible, ou simplement d'une " deuxième vie » dans autre monde, devient à un moment donné assez significative historiquement parlant. Pour le bonheur d'ailleurs des archéologues (et des archéoanthropologues) d'aujourd'hui qui, en dépit des pilleurs de tombes de tout temps, ont ainsi pu récolter une moisson d'artefacts qui font l'honneur (et le bonheur) des différents musées d'archéologie et d'histoire (sinon de l'art) sur tous les continents.

Mais attention! Ce que les tombes, les sépulcres, etc. ne nous démontrent pas, en tout cas pas au premier abord, c'est que l'histoire est aussi le témoignage de l'inégalité flagrante et arrogante. II n'existe guère un domaine qui expose, après réflexion, le plus crûment (et cruellement) les inégalités de classes ou, sans-façon, les positionnements sociaux inégalitaires que les pratiques qui entourent l'enterrement. Pour le riche et le puissant, ce sont des mausolées, tandis que pour le pauvre et l'ordinaire, c'est la fosse commune et la chaux. Rappelons, si besoin est, que les bâtisseurs des pyramides en Égypte (et au Soudan) ne sont pas enterrés dedans, et que les milliers d'esclaves, de travailleurs libres, d'artisans, d'artistes, de contremaîtres, de techniciens, d'architectes qui ont réalisé ces merveilles sont oubliés et rendus à la terre, sans aucune trace ! Et là, nous ne pouvons qu'évoquer le fameux (et si lucide) poème de Bertolt Brecht ${ }^{18}$. Or, devant la beauté et la majesté de l'éblouissement que nous ressentons au vu des Pyramides de Gizeh, il est aujourd'hui peu probable, pour le touriste ordinaire, que la condition ouvrière prenne la première place. Si les pyramides sont incontestablement des monuments mortuaires, elles représentent aujourd'hui des merveilles architecturales et "mystérieuses " que nous pouvons visiter et contempler dans toutes leurs splendeurs. La complexité de leurs constructions fait aujourd'hui rêver tant le simple touriste que les architectes chevronnés.

S'il s'agit de rêver, il y a lieu de mentionner le site funéraire du premier empereur chinois Qui Shihuangdi $(258-210 \text { a. J.C. })^{19}$. Les préchambres sur ce site nous ont révélé, après des fouilles commencées en 1974, des merveilles de l'art et d'artefact antique. On y a découvert toute une armée de fantassins, d'archers, de cavaliers tous en terre cuite polychrome ${ }^{20}$. Ce qui est ici magnifique, c'est que tout cela fût découvert dans la périphérie du site funéraire (qui totalise $56 \mathrm{~km}^{2}$ ) - à une distance de 5 kilomètres du tumulus central tandis qu'aucune fouille n'a été entreprise, ne fut-il que d'un pouce, en direction d'où se situait, pense-t-on, la chambre funéraire de l'Empereur. Et peut-être que c'est mieux ainsi, puisque ladite armée, qui avait traversé deux millénaires et plus sans trop de détérioration, s'effrite et se détruit aujourd'hui à un rythme galopant, comme c'est, hélas, souvent le cas avec tant d'autres merveilles qui nous ont été révélées par des fouilles et où la lumière, l'humidité ou les bactéries les réduisent en poussières. 
Loin de nous d'établir un quelconque catalogue des thanatopratiques puisqu'ils sont, répétons-le, trop variés, se conjuguent sur des variables culturelles qui changent et se modifient selon les époques et les cieux. Historiquement et culturellement, ils se distinguent et s'harmonisent suivant des croyances et des coutumes et se déclinent à l'infini. Notre attitude envers le cadavre et les restes humains est, insistons, humaine, trop humaine ! D'où nos attitudes, coutumes, traditions, usages et mœurs à l'égard du cadavre, en tant que manifestations de l'esprit de conscience des survivants. La façon dont nous traitons le cadavre se résume fatalement à l'idée que nous nous faisons de notre propre sort d'être humain.

\section{Une question de vie et de cadavre}

Enchaînons, maintenant, en affirmant que notre façon d'être devant le cadavre se confirme aujourd'hui le plus anthropologiquement possible dans le symbolique. En quittant l'archéologie et l'archéoanthropologie, nous entrons, de ce fait, dans l'anthropologie symbolique contemporaine où nous retrouvons le cadavre dans un dialogue entre le " refus culturel » (car le boomer autant que le post-boomer ne souhaite qu'être " jeune ») et une présence inévitable (car même les " jeunes" meurent). Le cadavre entre aujourd'hui symboliquement dans un jeu culturel du " reniement " et de la " dédramatisation ", où se mêle notre angoisse devant notre destin à tous et où se cache à peine un voyeurisme, soit-il esthétique, de bon aloi. Il convient, maintenant, de regarder de plus près, d'abord l'ambivalence de nos "minimisations et effacements ", avant de les analyser en tant qu'une nouvelle mystification « culturelle ».

Pour observer le phénomène invoqué de minimisation - effacement, il n'y a pas trop de chemin à faire, car cela se confirme une ou deux fois par semaine sur l'écran cathodique. La popularité, sinon l'engouement, qu'ont récoltés les séries de style hollywoodien traitant de l'industrie funéraire et le traitement des cadavres ces dernières années, ont de quoi nous étonner à première vue ${ }^{21}$. Rationnellement personne n'aurait pu prévoir cela ! Vu de l'extérieur, cela défie le bon sens. Les faits sont pourtant là : des séries télévisées sur les pratiques thanatologiques, sur le cadavre et les survivants, sans oublier celles sur la vie dans l'industrie des pompes funèbres, ont obtenu, dès le début du $21^{\mathrm{e}}$ siècle, un succès,

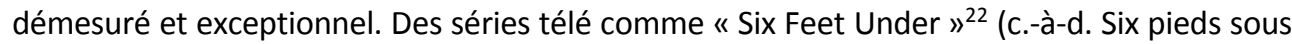
terre/ ou " en dessous " $^{23}$ ) ou encore " Dead Like Me ${ }^{24}$, "Bones ${ }^{25}$, tournant autour du cadavre, de la mort, de "l'existence " ainsi que des drames humains devant la dépouille d'un mort, en témoigne amplement.

Spécifiquement, la série "Six pieds sous terre " ${ }^{26}$ a tout pour soutenir nos propos, car elle représente, de façon emblématique, le prototype d'une télésérie culte captivante, centrée sur le cadavre ${ }^{27}$. La télésérie se focalise, en effet, sur une famille de croquemorts, embaumeurs, fossoyeurs et la dialectique complexe entre eux. Les télés narratives mettent en scène des cadavres "sans repos " qu'il faut " préparer ", réparer et maquiller, le tout, saupoudré par une dose d'humour supposément drôle et cassante, qui a tout pour fasciner 
les téléspectateurs. La famille vit, en quelque sorte, dans un genre de promiscuité étroit avec les cadavres, et toute l'originalité de la télésérie repose sur une double narration en parallèle entre la famille propriétaire (et leurs employés) et un cadavre, où s'entremêlent dramatiquement des survivants, des héritiers, des parents, des clients qui ont perdu un être cher. Ficelées avec maîtrise, ces histoires captent par leur crédibilité et leur réalisme, de même que par les enjeux psychologiques et existentiels fascinants qui s'y déroule, devant les yeux des téléspectateurs prêts à y croire sincèrement et à se laisser séduire.

II y a, de toute évidence, quelque chose d'attachant dans la narration de cette série et dans ses monologues, très élaborés, entretenus avec les cadavres, comme au-dessus d'un abîme où s'imite un "dialogue de transcendance ", un dialogue symbolique, où le téléspectateur est astucieusement invité, très subtilement, à se mettre, narrativement, à la place du défunt. Le cadavre devient ici le côté surnaturel de la vie et se conjugue, de ce fait, avec un aspect spirituel constant (sinon religieux et « chrétien »), sans jamais trop s'imposer avec la foi.

Si la mort rôde aujourd'hui autant autour de l'individu postmoderne que de l'individu moderne, la dialectique de " minimisation - effacement » culturellement en cours sert à l'individu contemporain à s'autoanesthésier. Autrefois, jusqu'à un temps historique tout récent, le cadavre était très près, terriblement près, de la vie quotidienne et prenait place dans les lieux communs d'habitation, dans la chambre à coucher de toute la famille. L'individu d'aujourd'hui, par contre, n'a plus à voir avec cette expérience et ne risque pas, non plus, d'enjamber un cadavre abandonné dans la rue la plus proche.

Si l'individu européen - pour ne parler que de lui - pouvait, jusqu'à une époque historique assez avancée - observer des condamnés à mort exécutés, et donc cadavre, suspendu dans des cages devant les portes de leurs cités, ou des personnes âgées, ou malades, mortes et en putréfaction avancée à l'intérieur de ces mêmes cités, pour ne rien dire des fossés communs extra-muros à ciel ouvert avec des cadavres en putréfaction répandant une odeur nauséabonde et de pourrissement (rappelant la vanité d'une existence éphémère), l'individu contemporain ne verra, à présent, rien de tel. À moins d'avoir de la malchance, il ne verra jamais un cadavre de toute sa vie ou alors, s'il le veut, il pourra voir un corps toiletté, maquillé et " arrangé ", dans un salon funéraire. Quant au cadavre des siens (famille, époux/épouse et enfants), il peut simplement refuser de les voir et quant à son statut de futur cadavre, il n'a qu'à penser à autre chose, rester " jeune " et refuser de dévisager son inéluctable destin. Somme toute, les cadavres que l'individu risque à présent de " voir " se trouvent plutôt, symboliquement, dans les jeux électroniques, dans les films de cinéma ou dans une série télévisée.

L'individu d'aujourd'hui n'a plus la même relation " naturelle " (sanitaire et hygiénique) ou " biologique " ou, de façon plus neutre, "coutumière ", à l'égard de la mort et du cadavre, qu'autrefois. Sous la forme d'une psychothérapie, il (ou elle) préfère regarder cela à la télé (rappelant nos propos sur " Six Feet Under ») et il (elle) préfère surtout la fuite en avant au niveau psychologique (pour ne rien dire de la fuite vers des pseudo croyances tricotées sur mesure pour célébrer l'idée futile du bonheur instantané) pour chasser toute onde non désirée et négative. 
L'individu contemporain a peur! Une peur bleue de mourir, peur devant les cadavres et devant l'image que cela lui reflète et surtout peur d'envisager son destin! Tout ce qu'il souhaite, c'est d'exhausser son angoisse, d'éloigner le vide existentiel qui le gruge et de tout faire disparaître dans sa négation. L'individu d'aujourd'hui cultive de préférence la croyance (ou l'attachement) à la " jeunesse » et se fige dans une " adolescence perpétuelle " ${ }^{28}$ qui n'a guère d'autre objectif que l'auto-illusion.

Le refoulement de la mort, en est un de la réalité qui attend l'humain et cela nonobstant les rêves imaginaires qui trottent dans la tête de l'individu. Quand il - l'individu ludique - se projette si désespérément dans des inventions artificielles pour éloigner les signes qui annoncent la mort, l'âge et la maladie, il vise aujourd'hui (comme jadis) une logique néo-païenne de "l'immortalité ", de revivre et de se "réveiller ". Autrefois, c'est la magie qui était mobilisée; aujourd'hui, c'est la science qui l'a remplacée. L'individu "sans qualité " (paraphrasant Robert Musil ${ }^{29}$ ) s'enrôle dans un désespoir d'une toute nouvelle mystification qui annonce, illusoirement, esthétiquement et de façon cosmique, sa survie. II s'agit (illusoirement) d'éloigner, à tout prix, l'image de la mort, de neutraliser l'idée de la mort au niveau du " corps", pour mieux se confirmer jeune et acteur d'une vie remplie de divertissements, de plaisirs et de jeux, le tout dans une logique d'adolescence perpétuelle. Nous avons donc, d'un côté, l'état d'adolescence et le plaisir du consommateur avide, tandis que de l'autre se trouve le refus de la réalité, de la vie et, en somme, le rejet d'envisager la mort comme un élément de notre condition humaine. II faut, au son de cette mélodie, refuser la vie pour mieux mourir.

Quand tout (surtout la réalité) devient un rêve illusoire, un refus de voir la mort, sa propre mort et celle des autres, comment réagir ? Quelle sera la réaction de l'individu devant les corps sans vie de ses grands-parents, de sa mère ou de son père, ou encore de ses propres enfants, pour ne rien dire de son épouse ou de son époux? Va-t-il se sentir embarrassé ? Gêné ? En total désarrois ? Peut-il éloigner ce corps inerte qui lui rappelle trop ce qu'il refuse de voir ? Faut-il qu'il se débarrasse de tout cela ? Comment se débarrasser de tout cela puisqu'il ne peut le jeter vulgairement aux rebuts ? Ou faut-il faire semblant, en ne suivant que des coutumes de bienfaisance, tandis que son âme est vide, son cœur sec?

\section{Le cadavre et l'humanité de l'être humain}

Le refus, si répandu aujourd'hui, de nier, d'éloigner, de banaliser ou encore d'instrumentaliser la mort, contamine notre relation avec le cadavre post-mortem. L'individu d'aujourd'hui n'ayant plus la sérénité d'accepter, comme autrefois, la mort, en tant que fin inévitable de toute vie, se révèle aussi, en règle générale, dépourvu de la force spirituelle qui s'explique à l'égard de la vie qui prend fin. En résultent un embarras, un malaise, un trouble qui n'éloignent pas la mort, car la mort arrive quand on s'y attend le moins. Quelques fois, au bout de la rue !

La mort ne se planifie que difficilement et, bien souvent, pas du tout ! Comment savoir si nous allons mourir comblés par la vie ou bien misérablement ? Comment savoir si un accident de bicyclette va nous tuer un matin d'avril, ou encore si une chute d'un 
escabeau fera de même en novembre ? Et tout le monde sait que la vie n'est guère une fête édénique et que les soubresauts de notre existence terrestre nous réserver bien des surprises. Nous ne vivons pas en tant qu' « île $»^{30}$, mais dans une société avec un corps et une psychologie plus complexe que nos idées fixes ou encore nos désirs avoués ou inavoués. La vie risque de nous jouer des tours en nous jetant dans le désespoir et le dénigrement, là où nous aurons, avec une force spirituelle, dû reconnaître la mort comme la ressource la plus profonde de notre existence.

\section{Cadavre, sépulcre et le non-objet interdit}

Nous l'avons suggéré auparavant, par l'éloignement physique et culturel, le cadavre devient en quelque sorte " tabou ». En d'autres mots, il devient un «non-objet » dont il faut s'occuper en tant que tel, dans toute sa singularité, avec soins et avec discernement. Apparaissent, en conséquence, des coutumes de sépulcre qui répond à ce statut particulier, à ce " tabou » et, surtout, qui insistent sur une obligation coutumière des vivants d'assurer un sépulcre pour un cadavre. Une telle tendance semble presque universelle, même s'il existe, comme toujours, des exceptions. Ce qui doit nous préoccuper par la suite, c'est de savoir comment la philosophie du droit a repris, formulé et problématisé cette obligation coutumière. Comment, en fin de compte, savoir ce qui est vraiment une obligation coutumière des sépulcres ?

\section{Le cadavre et la question de sépulture : Antigone et obligations coutumières}

Si le positivisme associe, métaphysiquement (sic !), l'existence d'une " obligation » avec la question du droit ${ }^{31}$, il ne s'agit que d'un faux pas, illogique et irrationnel, là où il aurait fallu admettre qu'une coutume est une coutume et, ici, qu'une obligation coutumière à un sépulcre représente ce que les mots en disent. II ne faut pas improprement parler d'un " droit » à la sépulture, car la question est toujours culturelle, circonstancielle, individuelle et partout dans le monde s'observe différentes formes de prises en charge des cadavres. Or, nonobstant toutes les variétés de ces prises en charge - pensons aux tours de silence des zoroastriens (correctement dit : mazdéens) mentionnées auparavant, aux incinérations du cadavre par le feu chez les hindous ${ }^{32}$, pour ne rien dire des différentes pratiques de sépulcres que nous trouvons dans les pays occidentalisés, il s'agit toujours de coutumes que nous observons et rien d'autre. En passant, il est à noter que le christianisme ne prône aucune obligation concernant le "sépulcre " et n'a en soi rien imposé concernant les pratiques relatives à la sépulture, sinon a accepté, repris et christianisé les coutumes déjà existantes dans tous les pays et cultures où il existe. Cela s'explique certainement par le fait que le christianisme n'est pas une religion de "l'extérieur » (de " soumission » ou d' " obligations »), mais une religion de "l'intérieur » (de l'espérance, de la foi, de l'amour, de l'engagement, etc.). Regardons maintenant de plus près, la question du respect des coutumes concernant le sépulcre. 
Mobilisons la pièce dramatique de Sophocle datant du $4^{\mathrm{e}}$ siècle avant J.-C et intitulée "Antigone $"^{33}$. Rien ne nous renseigne mieux sur la question de la coutume ancestrale, ancienne et "tribale » de l'obligation de sépulcre que cette pièce de théâtre. D'un côté, nous avons l'individu, les "proches", la parenté motivée par la piété à l'égard d'un corps mort, tandis que de l'autre, se trouvent l'État, la Souveraineté, le Prince qui suivent d'autres objectifs n'ayant rien à voir avec une telle piété. La popularité ou, simplement, la permanence de la figure de l'Antigone dans la pensée occidentale (et dans le droit nature ${ }^{34}$ ) nous témoigne de la leçon à suivre, qui est devenue emblématique pour la culture occidentale (et bien sûr aujourd'hui la culture universelle).

Rappelons succinctement l'intrigue de Sophocle : consécutive à une rébellion contre le tyran de Thèbes, Créon, le cadavre de Polynice gît mort devant le mur de la cité et est exposé aux oiseaux et aux animaux. Une sépulture digne lui est refusée par Créon, qui renforçant sa volonté par un édit, proclame la peine de mort pour quiconque s'occupera du cadavre du défunt. Antigone, la sœur de Polynice, refusant le commandement, ne peut accepter que l'on " abandonne sans larmes, sans tombe, une pâture de choix pour des oiseaux carnassiers " $^{35}$. Tandis que sa sœur Ismène prône de son côté la prudence devant les caprices du pouvoir, Antigone, quant à elle, agit et ensevelit le cadavre de son frère. Elle sera capturée par des gardes qui l'observaient ensevelir le corps mort dans le sable ${ }^{36}$ et " puis, levant un beau vase de bronze, elle couronna le cadavre d'une triple libation ${ }^{37} n^{38}$. Polynice, son frère, a, de ce fait, eu l'honneur de recevoir le rite de sépulture « afin de lui assurer auprès des morts un accueil honorable ». Antigone a accompli le devoir de sœur et de parente la plus proche. De ce fait, elle a autant occasionné une révolte contre l'ordre institué et contre l'édit, ou (improprement dit) le " droit positif ", qu'elle l'a défié en s'appuyant sur son propre jugement. Dans le drame de Sophocle, tout finit mal. Antigone se suicide et tous ceux qui chérissent Créon meurent avec elle, jusqu'au goût de vivre de Créon. Ce dernier constate que son édit et sa révolte contre les coutumes ancestrales des peuples helléniques n'ont provoqué que des dégâts et des malheurs. Chez Sophocle, c'est immanquablement les coutumes ancestrales qui gagneront et qui prouvent sa supériorité à l'encontre de l'hybris de l'homme, contre la rigidité anti-coutumière de Créon. Comme dans toutes ses pièces de théâtre, Sophocle s'affirme ici en vrai défenseur de l'ordre, comme il doit être et comme il a toujours été.

Il existe un mode, tout à fait postmoderne et irrationnel, d'attribuer une position de " droit » à chacun des différents personnages dans la pièce d'Antigone. II s'agit de confondre obligation coutumière et " droit », ce qui donne : une position de " droit » (c.-à-d. une obligation) coutumière (Antigone), d'un «droit » (c.-à-d. un édit ou acte législatif du pouvoir) positif (Créon), d'un « droit » en prudence ou encore d'une philosophie de droit naturel (Ismène). On retrouve donc la partisane des coutumes ancestrales, Antigone, qui tient fermement à les faire respecter, mais, surtout, à se soumettre à l'obligation que dictent les coutumes ancestrales. Antigone témoigne, en ce sens, au-delà de sa mort, qu'elle est « fidèle à [sa] race ${ }^{39}$. Quant à Créon, le Tyran, le représentant du " droit » positif, ou devrions-nous dire le " commandant » politique, il joue le rôle d'un véritable révolutionnaire qui, aveuglé par l'orgueil et le pouvoir, croit qu'il peut gouverner sans peuple, sans légitimité. Et Ismène, la représentante du droit 
naturel, fait ici figure de " prudence ", ne prônant ni le sacrifice au nom de la coutume, ni la révolution contre le pouvoir institué, pour que soient toujours privilégiés la sagesse, la prudence, la temporalisation, la non-confrontation, l'apaisement et le bon sens. Pour elle, le droit (naturel) représente l'art de naviguer entre Charybde et Scylla, pour mieux permettre aux passions (i.e. Antigone et Créon) de se réconcilier avec le raisonnable. Et si une telle navigation entre émotion et raisonnable se fait toujours au risque de se révéler vaine, ne s'agit-il pas aussi, chez Ismène, d'une constatation n'affirmant guère autre chose que des hommes qui se butent toujours à leur orgueil doivent, en fin de compte, idéalement se rattraper dans l'espoir d'une discussion raisonnable entre égaux?

Ce que nous démontre subséquemment la pièce de théâtre d'Antigone, c'est que les coutumes de sépulcres peuvent être modifiées et rationalisées. Nous ne sommes pas prisonniers de nos coutumes. La liberté vaut mieux que nos coutumes, aussi sacrées soientelles. Ce qui nous amène à l'institution sati chez les hindous, à savoir à l'obligation coutumière (et religieuse) pour une veuve de s'auto-immoler avec le cadavre de son mari ${ }^{40}$. Ce n'était pas une bonne coutume.

\section{Requiescat in Pace ou les profanations de cadavres}

Les profanations de sépultures ne sont pas un phénomène moderne et de loin. Les égyptologues sont sidérés de constater que les anciens sites archéologiques ont souvent été cambriolés à plusieurs reprises et avec des intervalles souvent éloignés l'une de l'autre. Le vol dans des sites de sépulcres est, en règle générale, facile, le risque de se faire prendre presque inexistant et le profit à en tirer quelques fois fabuleux. Mais si le vol est bien une façon de profaner un sépulcre, il ne détruit normalement pas le cadavre. Dans notre objectif, nous pouvons distinguer entre trois profanations du cadavre : profaner par haine, pour des raisons « idéologiques » ou pour s'enrichir.

En ce qui concerne la première justification de profaner un sépulcre, évoquons un évènement historique, tel que nous le rapporte l'illustre Cicéron :

"Sylla, vainqueur, fit disperser les cendres de Marius déposées auprès de l'Anio, poussé par une haine plus cruelle qu'il n'eut convenu s'il avait montré autant de sagesse qu'il montra alors de passion. ${ }^{41}$

La haine, la rancœur et l'adversité du sénateur et dictateur Lucius Cornelius Sylla (138 à 78 av. J.-C.) à l'encontre du général Caius Marius (157 à 86 av. J.-C) le suivra au-delà de la mort avec le refus de lui accorder un sépulcre, ainsi que par la profanation de ses cendres laissées au caprice du vent. De multiples autres cas de l'histoire nous témoignent le même phénomène et la même logique de haine. II s'agit d'effacer la trace de l'autre, de faire de l'autre un non-être et d'installer l'illusion qu'il n'a peut-être même pas existé ! Et si cela peut se faire avec des individus identifiables, cela peut également se faire à l'égard de grands segments d'une population comme nous le démontrent les totalitarismes de tout temps, d'hier comme d'aujourd'hui. 
Le deuxième exemple, la profanation pour des raisons idéologiques, se trouve dans le refus, le déni de sépulcre et se témoigne dans les idéologies totalitaires du $20^{\mathrm{e}}$ et $21^{\mathrm{e}}$ siècle. Ces idéologies totalitaires, dans leurs folies meurtrières, nous l'exemplifient tel que tout a été fait pour que les cadavres exterminés ne témoignent jamais et qu'aucun corps ne soit retrouvé. Les massacres des Arméniens en Turquie sont là pour nous illustrer ce fait, car de tous les 800000 à 1 million d'Arméniens atrocement tués par l'armée turque, par les milices et par les civils turcs, il n'y a presque aucune trace. Tout a été mis en œuvre pour que les cadavres disparaissent à jamais et pour ne jamais permettre à l'histoire de faire son jugement ; même s'ils se sont mépris sur ce dernier point ${ }^{42}$. Les nazis ont encore davantage perfectionné l'atrocité en faisant, surtout dans les années 1943 - 1945, disparaître leurs victimes, majoritairement juives, dans des fours à crémation, en espérant effacer toute trace et, surtout, des preuves. Les islamo-fascistes contemporains leur font présentement compétition en simulant les charniers de leurs carnages et abominations, en s'illusionnant que si rien n'a été vu, rien n'a été fait. Ce n'est en somme qu'une logique de perversion de " ni vu ni connu » où la disparation du corps-mort est un acte délibéré pour nier les faits, la responsabilité et l'histoire (et la mémoire) ${ }^{43}$. II est triste de constater qu'encore aujourd'hui, des personnes croient des menteurs.

Le troisième exemple de profanation de sépulcre, ce sont les voleurs de $\operatorname{corps}^{44}$ ( $d^{\prime}$ où s'insère notre première réflexion sur la nécropsie, l'examen post-mortem d'un cadavre). C'est avant tout une question de coutume (et de morale coutumière) et historiquement tardivement une question de législation politique. Au niveau coutumier s'observe donc l'interdiction antique et romaine d'autopsie et de dissection (anthroponomie). La même interdiction se retrouve dans les religions juives et mahométanes qui interdisent catégoriquement toute dissection. Par contre, le christianisme a eu une attitude plus ambiguë et complexe sur la question, en prônant à la fois le respect du cadavre, en même temps qu'il n'énonçait pas clairement une interdiction en ce qui concerne les autopsies et les dissections. Il s'agissait d'une question laissée aux " écrivains » et aux " autorités ", ce qui a engendré une ambiguïté historique (et bien sûr morale) bien connue, où l'accès au cadavre était pratiquement impossible officiellement, mais possible dans l'ombre, dans des laboratoires de recherche et dans les facultés de médecine. Historiquement, la culture médicale occidentale a largement profité de cette ambiguïté morale en ayant recours à des pratiques d'autopsies et des dissections selon le gré des chercheurs et des lieux d'enseignement de la médecine, et ce, depuis le début du Moyen âge. Mais il manque toujours de cadavres et l'histoire médicale occidentale, c'est l'histoire d'une pénurie et de vols (et d'achats) de cadavres.

Les voleurs de cadavres font le bonheur du folklore et des romanciers. Depuis le début du Moyen-âge, jusqu'à présent, les narrations sur les voleurs de cadavres qui les déterrent dans les cimetières pour alimenter le marché clandestin font rêver et frissonner.

D'un côté, on a le noble médecin qui a besoin d'un cadavre pour l'autopsier avec, pour objectif, d'acquérir plus de connaissances sur le corps humain, et de l'autre, un criminel sans scrupule qui, au profit de la nuit, déterre (et profane) un sépulcre pour s'en mettre "plein les poches". Sauf que le médecin de Frankenstein ne mérite peut-être pas la 
caractéristique de " noble médecin » et que le proverbe coutumier sur le recéleur et le voleur doit nous mettre en garde contre tout jugement péremptoire. II reste que les romanciers nous ont souvent donné des chefs d'œuvres sur le phénomène des voleurs de cadavre ${ }^{45}$.

Ce n'est que très tardivement, en règle générale à partir du $19^{\mathrm{e}}$ siècle, que le Législateur s'occupe de la pénurie de cadavres. Et il le fait dans des termes très généraux, en habilitant et en organisant. La législation prend la forme d'une protection générale de celui qui fait une autopsie sans pour autant se prononcer sur l'objet et la finalité de celle-ci. C'est un "marché » qui est régulé sans grande précision sur ce que constitue un cadavre ou encore une profanation. Le dernier peut être exemplifié avec « le mort fœtal » qui est bien un " cadavre " pour la mère qui attend son bébé et qui lui a donné son nom ! ${ }^{46} \mathrm{D}^{\prime}$ où le conflit moral de cette mère qui revendique son " cadavre " pour l'enterrer et le " trouble " qu'ont provoqué à plusieurs reprises les reportages journalistiques sur les "dépôts de cadavre de fœtus » dans des hôpitaux et des centres de recherches médicales.

Si certaines personnes sont tout à fait à l'aise de donner "leur " cadavre à une faculté de médecine, d'autres considèrent aujourd'hui l'État moderne et les hôpitaux comme des voleurs modernes de cadavres, qui trichent (en utilisant la Législation d'une façon stratégique pour obtenir des cadavres tels un réservoir d'objets biologiques de rechange) et qui mentent frauduleusement pour les obtenir. Nous reviendrons sous peu sur cette question, sous l'angle du « cadavre » en tant qu'objet de recyclage biologique.

Mais enfin ! Toute exposition d'un cadavre (et des restes cadavériques) n'est-elle pas, tout compte fait, qu'une profanation nonobstant la justification que nous voulons donner ? Tout musée qui expose un cadavre n'est-il pas " coupable » de troubler la paix éternelle ou, plus prosaïquement, le respect civilisateur que nous devons à celui qui est mort ? Et là s'observe que si le principe peut rassembler, la réalité et la pratique (surtout quand le cadavre devient objet d'art, de mythologie ou d'enseignement) divise et départage les opinions dans toutes les directions.

\section{Le cadavre mérite-t-il une sépulture aujourd'hui ?}

En fin de compte, existe-t-il toujours une obligation ressentie chez l'individu authentiquement postmoderne de fournir une sépulture à un défunt qui lui est proche ? Dans la tragédie d'Antigone de Sophocle, susmentionnée, c'était une évidence, quelque chose qui allait de soi, et cela a effectivement été évident historiquement, en général, jusqu'à aujourd'hui.

Or, justement, aujourd'hui, cela tient-il toujours ? N'existe-t-il pas, au contraire, une tendance post-culturelle (ou pro narcissique) qui dénie ou qui efface (anesthésie même) émotionnellement, culturellement et réellement le fait d'offrir une sépulture matérialisée d'une façon ou d'une autre à un cadavre ? Le nouveau « malaise dans la culture » n'est-il pas une pathologie contre-culturelle où le nouveau " Moi narcissique » n'arrive plus à avoir une relation réelle, affective et émotionnelle avec ce qui ne revient pas à "lui-même ", à ce « Moi » qu'il aime et qui lui rappelle inopportunément qu'il n'est pas vraiment né de la dernière pluie? 
II y a des raisons de croire que c'est une tendance contre-culturelle forte et appuyée, et que l'idéologie d'adolescence s'enkyste déjà profondément. C'est une idéologie de l'individu éternellement " jeune ", qui n'accepte pas le vieillissement et qui ne peut pas accepter non plus que quelqu'un meure. Dans un tel esprit tordu, existe-t-il un cadavre s'il n'y a pas de sépulture ? Et si le cadavre a été " spirited away ", enlevé sans que personne ne sache vraiment comment, et où il est passé, allant même à douter qu'il ait été là. Le narcissisme contre-culturel rejette en conséquence toute forme matérielle de la sépulture pour mieux nier le destin inévitable de tout individu. Avoir l'obligation de s'occuper et d'entretenir une sépulture pour les "siens" est devenue, pour beaucoup d'hommes d'aujourd'hui, un mauvais augure qu'il faut éviter à tout prix. Le plus commode, c'est de donner un contrat à une compagnie de pompes funèbres pour s'occuper de tout pour ainsi " tourner la page ". Bref, qu'il n'y ait pas de cadavres, de mort, mais uniquement le silence et le monde aussi aseptisé qu'un contrat sans âme, sans cœur, sans pitié ! On ne veut surtout pas voir l'image du mort.

Comprenons bien, devant ce refus culturel (ou encore émotionnel, ou pseudo spirituel régulièrement surchauffé artificiellement par différents récipients de sagesse orientale) de la mort, c'est simplement le refus du mort et de la vie qui s'exprime, et ce, de deux façons différentes: le refus de la mort comme destin de tout être vivant et la banalisation de l'existence, de notre existence, dans une " adolescence-isme » de bon aloi (et correspondant à la transformation dont nous avons parlé auparavant, de la société adulte en société « d'adolescentrique ») ; mais, aussi, un refus de la vie.

\section{Les transgressions, les hybris et la folie}

Logiquement, la suite de notre réflexion se concentre, à présent, sur le corps mort et les transgressions contre un cadavre ${ }^{47}$. Si le corps d'un vivant peut être (et peut devenir) l'objet d'abominations perverses, cela s'aggrave dramatiquement quant au cadavre. Une intrusion dans ce domaine, nécessairement succincte, n'a d'ailleurs rien pour assurer la tranquillité d'esprit d'une personne normalement constituée et lui montre un monde où la perversité se conjugue avec l'animalité, la folie, les pathologies (et les paraphilies) de tout ordre, où tout risque de se conjuguer à une démence n'ayant que l'apparence d'un être humain. C'est l'anthropophagie, la nécrophilie et la question de "nécessité », en tant que défense en droit pénal, qui doit nous intéresser ici.

\section{Anthropophagie et le cannibalisme par nécessité}

Il s'agit ici d'un topo classique en philosophie du droit ${ }^{48}$. Si classique qu'il y a certainement que très peu d'étudiants d'une faculté de droit nord-américaine qui n'ont pas abordé cela dans une classe de droit pénal ou d'introduction au droit ${ }^{49}$. Cela suit la suite 
logique et jurisprudentielle de " deux hommes et une planche de bois " seuls en mer après un naufrage et une planche de bois qui ne peut servir que pour une personne ${ }^{50}$.

En théorie juridique, c'est l'affaire judiciaire anglaise classique de $1884, R$. $v$. Dudley et Stephens ${ }^{51}$ qui attire notre attention. La Cour (High Court of Justice, Queen's Bench Division) était invitée à se prononcer sur le cas suivant : à la suite d'un naufrage, quatre membres d'équipage d'un yacht anglais, les Mignonettes, sont rescapés dans un bateau de sauvetage, à environ 1600 milles du Cap de Bonne-Espérance. Après quelques semaines à la dérive dans une zone peu fréquentée par d'autres bateaux, les ressources en eau et nourriture sont épuisées. La question de survie se pose : qui choisir en tant que personne à sacrifier pour la survie des autres ? Aucune décision ne fut prise et le plus jeune membre de l'équipage, un mousse de 17 ans, est tombé inconscient. Deux membres de l'équipage, Dudley et Stephens, décident alors de le tuer et de le manger (sans le consentement du troisième membre; ce qui nous permets de rappeler le proverbe : " qui ne dit mot consent »). Ils ont été secourus quatre jours plus tard. Ils ébruitent l'affaire eux-mêmes devant la commission maritime d'enquête, en se croyant couverts par les coutumes ancestrales relatives à la nécessité et la contingence en haute mer.

La Cour, ex adverso, a tranché en les condamnant, car la nécessité n'était pas, dans les circonstances, une défense concluante contre une accusation d'assassinat. Les deux accusés (car Brooke, le troisième dans le bateau, était uniquement convoqué en tant que " témoin ») ont, en conséquence, été condamnés à mort avec recommandation de clémence, et leur peine de mort a été commuée, par la suite, à six mois d'emprisonnement ${ }^{52}$.

Or, qu'est-ce qui représente la bonne procédure dans de telles circonstances ? Que tous se mettent d'accord pour participer ensemble pour prendre une décision commune ? Qu'il aurait été mieux, dans des cas semblables, de "tirer à la courte paille"53 ? Mais une telle question ne peut-elle être réellement résolue qu'à l'intérieur de la procédure égalisant tout projet de jugement ? $^{54}$ Or, ne s'agit-il pas, au contraire, de créer un abri circonstanciel contre toute incrimination en mobilisant l'image d'une " chance égale à tous " et d'une validité de procédure qui assure cette chance? La situation d'inégalité (i.e. "la folie » individuelle causée par la faim prolongée) est ici virtuellement supprimée ou, plus exactement, mise entre parenthèses par l'égalité d'une procédure acceptée et partagée au niveau des individus seuls. Si cela est jugé acceptable, la question judiciaire se concrétise-t-elle seulement dans la manière de choisir, de façon égalitaire, en ayant les mêmes "privilèges" ? Ce qui, soulignons-le, exclut le fait qu'une personne puisse choisir à la place d'une autre, mais aussi que tous puissent, si possible, partager le même privilège de survie, pour se justifier de commettre un assassinat, un acte de cannibalisme.

Quand toutes les personnes ont droit à un privilège égal, personne n'a de quelconque privilège ! C'est uniquement à partir de ce moment-là que la procédure de sélection va viser à "choisir" la personne qui sera "sacrifiée", et donc celle qui sera "privilégiée" survivra. Car, c'est l'individu et lui seul qui, par engagement dans la procédure, devra choisir son destin. Si dans la société politique, le "préalable" renvoie aux « textes législatifs " agréés publiquement en tant qu'institutionnellement valides, le seul préalable dans l'affaire $R v$ Dudley et Stephens était l'égalité des chances et de privilèges. 
Une telle procédure de sélection annihile surtout le soi-disant "droit du plus fort" (c.à-d. le privilège de pouvoir et de l'autorité) au profit de la conception selon laquelle les privilèges individuels (et que les auteurs de doctrine juridique appellent « un droit " ou " des droits ») sont précisément le résultat d'une procédure au cours de laquelle nous pouvons juger juste, impartialement et adéquatement. Une personne n'est pas l'égal de l'autre si elle ne peut, par ses propres actes et moyens, en toute autonomie, se confirmer en tant que tel. II n'existe pas d'égalité lorsque celle-ci est donnée ou accordée par une loi ou une Constitution, car une égalité donnée c'est une inégalité confirmée.

Le cas de $R$. v. Dudley et Stephens ne révèle aucune perversité ou paraphilie. Le cannibalisme en tant que perversion, en tant que "volonté " (de chair, de puissance, etc.) nietzschéenne ou schopenhauerienne, peu importe, existe pourtant ! Deux célèbres cas peuvent être évoqués : le cannibale de Milwaukee ${ }^{55}$, ou encore le cannibale (également sous la forme d'une homosexualité singulière) d'Allemagne ${ }^{56}$.

Le premier cas que nous examinons, le cannibale homosexuel de Milwaukee, a largement révolté les esprits aux États-Unis dans les années 90. Jeffrey Dahmer a tué dixsept jeunes hommes (et peut-être plus) entre 1978 et 1991. Il les a violés avant de les tuer pour ensuite les garder en souvenir, les démembrer et pratiquer la nécrophilie et le cannibalisme. Les psychologues diagnostiquent une multitude de paraphilies, comme agalmatophilie (ou pygmalionisme), fétichisme, hébéphilie et exhibitionnisme, de même que la nécrophilie et le cannibalisme. Quand il fût arrêté par les policiers, ces derniers ont trouvé, dans son réfrigérateur, des parties corporelles destinées à être mangées, mais qui jouaient également un rôle pathologique au niveau du fétichisme et de l'agalmatophie ; les parties corporelles lui permettaient de revivre l'expérience de l'avant-assassinat, l'assassinat et l'après-assassinat, comme un moment d'excitation et de satisfaction. Aux États-Unis, Jeffrey Dahmer a aujourd'hui acquis le statut «culte » dans (et en faveur de) la contre-culture " métal », " alternative ", " gay », etc. ${ }^{57}$

Le deuxième cas à examiner vient d'Allemagne et concerne le crime d'Armin Meiwes (le cannibale de Rothenburg). C'est un crime de cannibalisme et une excellente intrigue judiciaire, car Meiwes avait tout prévu pour éviter d'être incriminé et jugé. Le cas commence par la publication de plusieurs annonces sur Internet sollicitant des volontaires pour se faire manger physiquement et corporellement. Armin Meiwes a reçu 430 réponses positives à sa sollicitation et après des négociations - et des retraits - son choix se fixe sur un ingénieur de quarante-deux ans. Après une relation homosexuelle, ils décident ensemble de couper le pénis de la victime et de le manger ensemble. Celui-ci dégusté, ils décident, de concert, qu'Armin Meiwes «tue " l'autre personne, sa victime, ce qu'il fait par la suite, sans pour autant que toutes les modalités arrivent à être prouvées et, surtout, sans que les actes précis de la victime puissent être discernés clairement. L'acte de « tuer » était expressément mis en scène pour échapper à toute incrimination (et filmé pour servir de "preuve " dans un procès criminel prévisible). II semble, plus précisément, que la victime s'intoxique et se lacère pour se vider entièrement de son sang (ce qui suggère un suicide) pour, le lendemain, recevoir un " coup de grâce » d'Armin Meiwes. Or, impossible de prouver que la victime 
n'était pas morte à ce moment-là (et à cause de son suicide), ce qui aurait restreint l'acte d'accusation à une " profanation de cadavre » ou, encore, à une atteinte à la " dignité d'un individu mort ». Autrement dit, des actes de cannibalisme (ce qui était l'objet de sa sollicitation), car Armin Meiwes a coupé 30 kilos de viandes du cadavre, qu'il a consommé tranquillement les jours suivants. La suite de l'histoire, c'est qu'il a été arrêté par la police et qu'il est passé aux aveux. Mais comment l'incriminer ? La stratégie d'Armin Meiwes (ou plutôt de ses avocats) a été de plaider un « homicide ", car il a été présent durant toutes ces étapes et donc, criminellement responsable. La stratégie de plaider un " homicide " a été très payante et ne lui a donné, en $1^{\text {re }}$ instance, que huit ans et demi de prison en 2004 . Mais après une critique virulente de la population et à la suite d'un pourvoi en cassation, le jugement final a déclaré qu'il s'agissait là " d'un assassinat à caractère sexuel ", ce qui lui a valu la réclusion à perpétuité ${ }^{58}$.

\section{Éros et thanatos : la nécrophilie}

À côté du cannibalisme, le plus sombre « secret » est certainement la nécrophilie59, ou le fait de se procurer un plaisir sexuel avec un cadavre. II y a une relation obscure entre Éros et Thanatos60. C'est le côté sombre de la " chambre des morts " qui attire les nécrophiles avec, surtout, le plaisir de violer, symboliquement et réellement, " un cadavre qui présente la forme humaine avec une absence totale de volonté et qui, par ce fait même, est capable de satisfaire le besoin morbide de subjuguer d'une manière absolue et sans aucune possibilité de résistance, l'objet désiré. "61 La règle coutumière, autant en Europe qu'en Moyen-Orient, était, en conséquence, qu'un cadavre (et surtout le cadavre d'une femme) devait être enterré rapidement (dans un délai de trois jours) et être surveillé par la famille (et surtout par les femmes de la famille) jusqu'à l'enterrement. Ceci pour contrecarrer tout abus sexuel sur le cadavre. Mais avec l'apparition de l'industrie des pompes funèbres, de telles coutumes deviennent inopérantes et la question de confiance peut, ainsi, être trahie.

Il y a ici de vraies histoires, comme des moins vraies, que nous utiliserons pour une fiction littéraire et cinématographique! II y a lieu de commencer avec le film vedette "Kissed $\|^{62}$ qui met en scène une jeune femme qui aime les cadavres et qui aime faire "l'amour » (sic !) avec. Plus concrètement, elle profite de la rigidité cadavérique de l'organe masculin pour s'y faire, sans plus de détails de notre part, cavalière. C'est à la fois un film fascinant (car extrêmement bien fait), un film scabreux (par son thème et par la froideur clinique de l'approche cinématographique) et un film de conte romantique et relationnel (par l'ambiguïté sexuelle de la jeune fille et par l'effet d'attirance que cela produit chez son prétendant Matt, qui essaie de la suivre dans son univers). Le film a obtenu un statut de " culte underground " pour les aficionados, même si c'est injuste de le réduire au macabre ou encore à la transgression, là où une approche psychologique semble plus appropriée et pertinente. $\mathrm{Vu}$ en tant que syndrome " psychologique ", le film est simplement une mine d'or du labyrinthe psychique de la jeune protagoniste Sandra et de l'impossibilité qu'elle semble ressentir face à la construction d'une relation sexuelle saine à l'égard d'autrui. Le 
film est précurseur en ce sens qu'il met le doigt sur le fait de voir comment " le peu chaud » devient un obstacle psychique pour un épanouissement sexuel et psychologique de qualité. Enfermé dans son intimité, dans son "identité ", comme le sont de plus en plus de contemporains, la jeune fille ne communique pas avec sa propre sexualité ni avec la sexualité d'autrui, et tout se ferme dans la paraphilie ou encore le fétichisme sexuel et dans le blocage face à la chaleur humaine. Et là où il manque de chaleur humaine, c'est la froideur de l'objet, la froideur d'un membre masculin appartenant à un cadavre, qui sert de remplacement, voire de substitut. II s'agit, plus encore, d'un objet qui, comme tout objet, peut procurer du plaisir ou même l'extase (comme l'indique le titre québécois de ce film), mais au coût de l'aliénation sexuelle et humaine.

À côté du film, il y a la réalité et, surtout, l'incrimination pour " profanation de cadavre " ou l'acte de nécrophile consistant à utiliser un cadavre pour sa gratification sexuelle. Ce sont surtout des corps morts de femmes dont il s'agit et aussi, presque exclusivement, après que la personne ait tué la femme en question pour se servir de son cadavre afin de se satisfaire sexuellement. C'est en fait un viol abominable, car l'assentiment est impossible, voire sans objet ! Au niveau de l'incrimination, les affaires de ce genre sont plutôt rares (heureusement), car la plupart des meurtriers, même les plus endurcis et brutaux, s'arrêtent devant un tel acte. Or, au niveau de la paraphilie, il faut avoir une faille psychologique bien particulière pour s'engager sur un tel chemin.

\section{Le cadavre et les limbes de l'objectification}

Est cadavre ou n'est pas cadavre, telle est la question ! Réécrivant ici librement les fameuses paroles de Shakespeare, de sa pièce "Hamlet ", cela n'est guère une question de style littéraire. En fin de compte, qu'est-ce qu'un cadavre aujourd'hui ? Une matière, un objet à recycler au profit d'un autre objet, un autre sujet ? Un objet destiné à être identifié pour notre bénéfice, pour nos propres intérêts, pour notre bien ? Nous avons aujourd'hui des cadavres qui se refusent un sépulcre, des cadavres qui seront honorés comme des objets d'art, des cadavres qui accouchent des bébés, et nous avons, enfin, des politiques étatiques et médicales qui nous incitent à donner notre "cadavre ", comme matière brute pour la réparation biologique d'autrui. Regardons donc de plus près cette problématique.

\section{Cryogénisation : ou le désir du mort d'être vivant}

Dans notre société " adolescentrique ", mieux vaut être éternellement jeune, désirable et beau/belle. Quand on est mort, cette partie est réduite à néant ! Qui a envie qu'une aventure " adolescentrique " prolongée finisse de cette façon-là ? Personne. II faut, en conséquence, tromper la mort, voilà le credo de l'adolescentrisme! Durant " la vie ", il s'agit de repousser tout signe (et toute parole) évoquant la mort. Ce n'est qu'après, au seuil de la mort ou du suicide, qu'il faut opter pour la cryogénisation dans l'attente d'être un jour 
réveillé pour une seconde jeunesse ${ }^{63}$. Étant entendu que, dans le futur, il y aura des remèdes pour guérir les maladies et les dysfonctionnements biologiques qui nous hante aujourd'hui.

La quête d'immortalité a, semble-t-il, toujours hanté l'humanité ${ }^{64}$. Dès les procédés de momification (qui était une technique de préservation du corps), aux espoirs mis dans les différentes formes de l'alchimie, de la science (et de la pseudoscience), l'idée de repousser ou de supprimer la mort a longtemps fasciné l'esprit humain. Hélas, en vain ! Or, tout semble (enfin !) se bousculer avec la cryptogénétique, avec l'idée de sauvegarder (en principe) le corps entier pour une éventuelle " réanimation », une éventuelle deuxième " vie », toujours sans sépulcre ${ }^{65}$.

L'idée de la cryptogénétique peut paraître assez simple, mais elle est extrêmement compliquée, sophistiquée et coûteuse. Pour donner un aperçu succinct de la méthode, nous pouvons dire qu'il faut arriver à planifier cela bien à l'avance, pour avoir un résultat satisfaisant. Le mieux c'est, en fait, qu'un expert en cryogénisation accompagne le mourant (et idéalement le suicidaire) avant la mort, pour mettre en place les différents procédés appropriés pour les parties distinctes du corps. L'expert en cryogénisation intervient le plus rapidement après la mort (idéalement après, ou au moment d'une crise cardiaque), pour " nourrir » le cerveau, afin d'en permettre " sa survie ». Pour cela, il lui fournit un apport en oxygène et en éléments nutritifs. Après, il amène rapidement le corps à $\sim 0^{\circ} \mathrm{C}$. Pendant ce temps, une machine " cœur-poumon » entretient l'alimentation et la circulation du " sang " (afin qu'il ne coagule pas et qu'il ne se cristallise pas) du nouveau mort. Ensuite seront injectés des médicaments ayant pour but de réduire le métabolisme naturel du corps et le sang sera remplacé par une solution liquide qui empêchera la coagulation et le développement de bactéries. Cette solution a également le rôle "d'antigel" pour le corps. Puis le corps est entreposé dans une capsule d'azote liquide à la température de -196 C., ce qui permet de garder intactes les structures cellulaires et, ainsi, de contrer l'usure et le vieillissement ${ }^{66}$. À la fin, il ne s'agit plus que de rester congelé pour quelques siècles ou quelques millénaires et d'attendre, avec patience, dans un Cryotorium ou ailleurs ${ }^{67}$. Si tout marche bien, quelqu'un sera là pour s'occuper de vous !

En fait, le cadavre se trouve littéralement " suspendu dans sa mort », suspendu dans l'attente d'une éventuelle (et heureuse!) réanimation dans le futur pour recevoir un traitement médical plus avancé et susceptible de vaincre la maladie (ou le dysfonctionnement) qui avait initialement affecté la personne avant sa mort. Donc tout l'espoir, pour le cadavre et ses survivants, est investi dans la " science médicale " à venir, qui sera plus avancée qu'aujourd'hui. C'est l'avenir qui décidera, en fin de compte, si la méthode est adéquate ou s'il ne s'agit que d'une hyperbole scientifique ou, en d'autres mots, un échafaudage néopaïen. Et le paradoxe est tel que si personne ne peut prévenir l'avenir, c'est la dernière solution qui, scientifiquement parlant, se désigne comme la plus probable. Mais peut-être que les cadavres cryogénisés seront, à l'avenir, ce que les momies sont pour nous aujourd'hui !

En tout cas, ce qui est vrai, c'est que personne n'a refusé à ces individus un sépulcre. C'est eux qui n'en ont pas voulu, car ils ne voulaient pas être " mort » ou, du moins, de ne l'être que " temporairement »! En fait, c'est l'individu qui effectue une objectification de soimême, qui se forge un espoir d'immortalité sur l'axe de l'objectification de son corps, de son propre cadavre. 


\section{L'art, ou la beauté du cadavre.}

L'art avant-gardiste et " progressiste » contemporain n'est pas uniquement réductible à son cul-de-sac " adolescentrique "; c'est aussi le lieu où nous trouvons les adorateurs de "l'art pour l'art ». Comprenons bien : ce qui compte, pour l'heure, ce n'est pas l'art (et surtout pas la beauté trop difficile à atteindre aujourd'hui), mais la capacité pour l'artiste de devenir lui-même un objet à vendre, de devenir la marchandise de lui-même, de devenir l'individu qui a mérité "ses quinze minutes de célébrités " et qui vend la "marchandise " comme venant de lui. Puisqu'il est extrêmement difficile dans une telle situation d'inventer quelque chose de vraiment nouveau, tout le monde a déjà copié ce que les autres ont fait, il n'est pas surprenant que les artistes arrivent aux cadavres. Pourquoi ne pas utiliser des cadavres comme de l'« art ", pourquoi ne pas exposer artistiquement des cadavres? II y n'a qu'un obstacle mineur qui doit être surmonté : présenter cela sans détruire le cadavre. C'est ce qu'ont résolu un artiste et l'anatomiste Gunther Von Hagens, avec sa technique de " plastination ${ }^{68}$ des cadavres. Le succès de ses expositions des cadavres plastifiés - 30 millions de spectateurs en 2017 et cela augmentent sans cesse - en témoigne ${ }^{69}$.

L'idée n'a rien de nouveau, tout le monde sait que les momies égyptiennes ont pendant des siècles été exposées comme des œuvres art. C'est souvent dans les Musées d'art que nous les retrouvons, par exemple, aux États-Unis. Et le philosophe anglais du droit Jeremy Bentham (1748 - 1832), qui croyait fièrement léguer son corps mort à la " science ", serait certainement déçu et contrarié s'il apprenait qu'aujourd'hui, son cadavre a rejoint le cabinet de curiosité pour voyeur averti. II s'agit en fait d'une " maquette " qui est exposée pour le plaisir des curieux ${ }^{70}$. Günther Von Hagens, par contre, s'engage plus résolument sur le marché du voyeurisme avec son procédé de plastination, car il promeut, prétend-il, la science et la pédagogie scientifique (rien de moins !) en se servant de la beauté du cadavre en tant qu'art. S'il expose en tant qu'artiste et si l'étiquette de précurseur artistique postmoderne lui fait éloge, il se vante lui-même d'être un scientifique, loin de l'idée d'être un marchand d'expositions de cadavres maquillés "scientifiquement ". Or, derrière la façade, c'est pourtant un business multimillionnaire avec des expositions concourant simultanément sur cinq continents et dans une multitude de pays qui se révèlent. Les shows de Gunther Van Hagens (ou plutôt les directeurs de l'entreprise du même nom) ressemblent aux fameux " freaks-show » du $19^{\mathrm{e}}$ où la curiosité et la fascination des consommateurs semblent identiques. C'est uniquement les voyeurs du $21^{\mathrm{e}}$ siècle qui ne comprennent pas la ressemblance frappante avec ce qui s'est fait au $19^{\mathrm{e}}$ siècle. La différence, paradoxalement, c'est uniquement que la technique et l'art de Gunther Van Hagens, en ce qui concerne la préservation des cadavres, sont indubitablement de meilleures qualités.

En fait, si nous acceptions que les cadavres de Gunther Van Hagens soient de l'ordre de l'art, Marc Jimenez n'a-t-il pas raison d'écrire que : « Sans doute faudrait-il aussi dénoncer cette autre tartufferie qui consiste à s'offusquer des aspects morbides, scatologiques, abjects, infâmes, mortifères de certaines productions actuelles, tout en acceptant sans sourciller le voyeurisme indécent auquel incite l'exhibitionnisme souvent obscène des médias et de 
l'industrie culturelle. Mais les plastinats de Von Hagens font partie de ces cas limites, ceux pour lesquels la frontière est difficile à établir entre art et non-art, entre provocation gratuite, exhibition sadique et mise en scène dénonciatrice de l'horreur. Seul un débat esthétique argumenté, contradictoire et, si possible, public permettrait d'en décider. " $^{71}$

II est peu probable qu'un tel "débat esthétique argumenté » aura lieu, le milieu artistique d'aujourd'hui est trop fort et n'y voit aucun intérêt (surtout pas pécuniaire). La version la plus réaliste reste de croire que le « voyeurisme " s'installera en douce. La foire aux cadavres (et notre voyeurisme) fera tout naturellement, aujourd'hui, partie de la vie des citadins du monde. Chasser le cadavre, il revient en version plastinat !

\section{La couveuse : le cadavre pour produire des bébés}

Aldous Huxley avec son roman d'anticipation nous avait avertis ${ }^{72}$ : pourquoi ne pas remplacer les mères avec des couveuses ou mieux avec des cadavres-couveuses ? Pourquoi ne pas (comme le revendiquent les "féministes " anti-mères et anti-sexe) libérer une fois pour toutes les femmes de leur capacité de faire et de porter des bébés et de concéder tout cela aux cadavres-couveuses ? Pourquoi ne pas réutiliser les cadavres des femmes (surtout de jeunes femmes) en tant que couveuses ? Cela épargnerait les vivantes et ce sera, dit-on, utile à tout le monde, surtout au niveau de l'économie et du pouvoir féministe.

Si cela avait pu encore sembler utopique quand Huxley écrivait son roman, il s'agit maintenant d'une réalité, tel que nous le démontre l'histoire du cadavre qui donna naissance à un petit garçon paru dans le journal Le Monde (Paris) le 6 août 1993, sous le titre "Un bébé naît 104 jours après la mort de sa mère » :

Une équipe de médecins californiens a réussi à faire naître le bébé d'une femme enceinte, cent quatre jours après avoir déclaré cette femme cliniquement morte. « Il s'agit d'un beau bébé de sexe masculin parfaitement développé ", a déclaré le docteur Richard Fürth, qui a surveillé la césarienne effectuée par ses collègues au Highland General Hôpital d'Oakland (Californie).

Le bébé, qui pèse 2,2 kilos, devrait survivre malgré certains problèmes respiratoires. La mère, Trisha Marshall, âgée de vingt-huit ans, avait été déclarée cliniquement morte alors qu'elle était enceinte de dix-sept semaines, deux jours après avoir reçu une balle dans la tête au cours d'une tentative de cambriolage.

Une équipe médicale a maintenu la femme artificiellement en vie, afin d'assurer le développement du fœtus, et ce à la demande de sa famille et d'un homme qui affirmait être le père de l'enfant. Le système de survie a été débranché après la césarienne, a-t-on indiqué de même source. Selon les médecins, seul un autre fœtus avait survécu davantage, il y a quelques années, dans l'État du Vermont, une femme avait été déclarée cliniquement morte dans la seizième semaine de sa grossesse. ${ }^{73}$

Vu que la femme était cliniquement morte, c'est, de ce fait, un cadavre qui a accouché d'un petit garçon. On a intentionnellement utilisé le cadavre de la femme pour obtenir un être vivant. La seule raison pour laquelle le bébé est né, c'est parce que les 
médecins ont utilisé le cadavre de la mère comme couveuse. Que le journal n'ait pas jugé bon ici d'utiliser le mot "cadavre " ne change rien à ce fait, mais montre notre désarroi devant ce que nous prouvent les faits : à savoir qu'un cadavre a été utilisé comme couveuse pour produire l'objet (sic !) ou ce qui est le plus convoité sur notre terre : un bébé.

II n'est pas inutile d'examiner également le commentaire scientifique qui a accompagné cet article de presse :

Les progrès de la réanimation autorisent aujourd'hui l'accomplissement de tels « miracles ». II y a quelques mois déjà, une affaire similaire avait alimenté une très vive polémique outre-Rhin lorsque des médecins bavarois avaient maintenu en " survie " artificielle une jeune femme enceinte âgée de dix-huit ans. L'enfant n'avait pu voir le jour, un avortement spontané s'étant produit six semaines après le début de la réanimation.

De telles affaires voient s'opposer avec force les tenants du respect absolu de la vie dès la fécondation et ceux qui dénoncent ce qui constitue une nouvelle forme d'acharnement thérapeutique. L'une des questions essentielles posées est celle de l'impact que peut avoir une telle situation sur le développement du fœtus et le psychisme de l'enfant. L'utérus d'une femme " cliniquement morte " peut-il raisonnablement être assimilé à une " couveuse » ? Qu'en est-il des relations si importantes normalement établies entre la mère et l'enfant qu'elle porte ? La science et la médecine sont, ici, muettes. ${ }^{74}$

Et si la science et la médecine sont muettes, elles ne sont pas les seules. Devant le miracle que constitue tout nouveau-né qui rejoint l'humanité, il y a des raisons de se taire. Mieux vaut tout dire par le silence, par l'émerveillement d'un bébé vivant. Et après le silence, c'est la douce mélodie d'un enfant qui, seule, s'impose.

Mais cela ne devrait pas pour autant nous empêcher de nous poser des questions, de nous interroger sur nos techniques relatives aux " cadavres-couveuses » et sur notre futur, à savoir la permanence et l'industrialisation des "cadavres-couveuses " (déjà possible théoriquement à partir d'une implantation in vitro), pour produire ces bébés tant désirés. Et, au-delà de la technique, que penser du développement du fœtus et le psychisme de l'enfant produit désormais par un "cadavre-couveuse " ? Si la mère est un "cadavre-couveuse ", comment se développe le psychisme de l'enfant? En fait, nous n'en savons rien, car ici s'ouvre une porte vers un paradis (ou un enfer !) où il n'y a désormais pas de vraie mère, sinon un cadavre qui accouche pour des détenteurs futurs de l'autorité parentale. C'est une nouvelle expérience et le " meilleur des mondes " qui nous attend si nous le voulons ou, de façon plus probable, si nos élites le souhaitent !

\section{Le cadavre en tant que source d'organes pour autrui}

C'est de convenance d'aborder la politique du « don d'organes » en tant qu'amour du prochain, de l'aide à celui qui en a besoin ou, encore, comme l'ultime geste humain à l'égard d'autrui. La litanie de notre idéologie contemporaine, c'est de mettre en avant le «don 
d'organes » en tant qu'acte noble, un acte " d'amour », un acte altruiste que concède une personne vivante ou, après la mort, qu'autorisent les parents proches. Mais cela peut aussi être construit sur une présomption (construit d'une manière moraliste) selon laquelle la personne souhaitait faire don de ses organes à sa mort. À ce moment-là, la loi autorise les médecins à utiliser les organes après la mort d'une personne. Et c'est aussi bien un acte qui peut être fait du vivant de la personne, en donnant un rein par exemple. Contester toute cette bienveillance idéologique n'a aucun sens. L'utilitarisme, c'est l'idéologie de note époque. Et le cadavre représente bien une source d'organes, presque infinie, qui peut servir à autrui.

C'est le médecin moderne qui permet le remplacement et la substitution des organes humains. Avant le 20e siècle, il n'était pas possible de transférer (ou de greffer) un organe humain à un autre corps humain. Tu vivais avec le corps que tu avais et tu mourrais avec. Aucune possibilité de vaincre le "rejet biologique" (et immunologique) à l'égard d'un organe étranger et aucun traitement immunosuppresseur (normalement à prendre pendant toute la vie) n'était disponible. Tout cela a changé au cours du 20e siècle où se gagne, étape par étape, organe par organe, la possibilité d'échanger ses organes pour des nouveaux. La première transplantation cardiaque a été réalisée en 1967 par le professeur Christiaan Barnard, en Afrique du Sud. Elle a représenté autant un succès emblématique, qu'un symbole que le temps avait vraiment changé ${ }^{75}$. Désormais, $c^{\prime}$ est devenu possible. Et c'est un moyen pour vivre, vivre plus longtemps et en bonne santé.

On constate que le domaine des "transplantations", des " greffes " et la question des bénéficiaires sont aujourd'hui entièrement légiféré (dans le monde occidental). II s'agit certainement d'un des domaines législatifs où il y a le plus de textes contraignants, dirigistes et habilitants. C'est un domaine à coup sûr hyper-légiféré ! Tout (ou presque) a été légiférer. Et la raison tient certainement du fait que cela touche à la vie, mais aussi à la mort. On se retrouve face à des questionnements éthiques (et de " justice ") où il n'y a pas de consensus existant et où il serait invraisemblable de l'obtenir. Et là où l'éthique ne peut rien faire, c'est la politique qui le supplée et qui instaure, par la législation, des modus vivendi acceptables pour le plus grand nombre.

De ce fait il existe, surtout dans le monde occidental (et bien évidemment extraoccidental), des corpus substantiels de textes législatifs et réglementaires, de même que des " directives » hospitalières, médicales, épidémiologiques et hygiéniques concernant la transplantation des organes et la question du choix des bénéficiaires. Il existe aussi, au niveau international, tout un corpus conventionnel (et policier) pour lutter contre le trafic des organes humains ${ }^{76}$ notamment issus d'un cadavre. Et là où il y a un marché, il y a aussi des personnes qui veulent en profiter, en achetant et en vendant des organes issus d'un cadavre. Et si l'État (et les Associations/Sociétés nationales de transplantations d'organes) peut récolter des "organes humains" presque librement et à l'abri d'une législation habilitante, les individus "privés » ne peuvent le faire qu'en achetant des cadavres ou des morceaux " tout frais " auprès des médecins, des infirmiers, des hommes et des femmes du monde hospitalier, sinon des croquemorts et des embaumeurs modernes. 
D'où la question de savoir si, au lieu d'incriminer la vente des organes humains issus des vivants ou des morts, il ne faut pas plutôt la rendre légale afin de pouvoir évaluer, sans préjugé, si cela ne permettrait pas d'arriver à surmonter la pénurie d'organes ainsi que les problèmes hygiéniques et médicaux issus de l'incrimination de cette pratique. L'article de Becker, Gary S. ${ }^{77}$ et Elias Julio "Introducing incentives in the market for live and cadaveric organ donations"78 (" Présenter des incitations sur le marché des dons d'organes vivants et cadavériques ») a été programmatique pour une telle position politique. Gary S. Becker et Elias Julio prônent fortement que l'établissement d'un marché libre des « dons d'organe » vivants et cadavériques pourrait aider à résoudre le problème d'une pénurie de transplantation d'organe. Le fait d'autoriser la vente libre d'organes permettrait de supprimer le marché noir et les vols d'organes cadavériques, de même que d'améliorer la qualité sanitaire des organes pour une transplantation. Leur modélisation économique, au moment de la rédaction de leur article en 2007, a permis d'estimer le prix des reins humains (15000\$) et le foie humain (32000\$).

La position de Gary S. Becker, associée au courant du "droit et économie ", a été politiquement reprise par la philosophie libertarienne, surtout en Amérique du Nord. L'interdiction de la vente d'organes est, pour la majorité des libertariens, une atteinte à la liberté de chacun de disposer de son corps. Les lois qui punissent la vente d'organes sont, selon eux, des lois illégitimes au regard des « droits naturels » (i.e. la liberté fondamentale) des individus.

La même forme de raisonnement a été formulée concernant le corps des condamnés à mort. Dans une éthique de "repayer à la société ", plusieurs théoriciens ont évoqué l'idée que le corps des condamnés à mort soit disposé en faveur des personnes malades ayant besoin d'une transplantation d'organe pour survivre. L'idée est, de toute évidence, que si leur vie à servir la mort, leur mort peut maintenant servir à la vie. Mais cette idée est ici, surtout, circonscrite à l'intérieur d'une politique officielle et publique et ne s'aventure pas dans des trafics privés et douteux. À cet égard, il convient de mentionner que certains états asiatiques sont soupçonnés de favoriser et d'utiliser, de façon tout à fait immorale, le cadavre (des peines capitales) pour alimenter un trafic d'organes qui profite, en premier lieu, aux caciques du régime en question.

Le don d'organe d'une personne morte est, finalement, une politique du cadavre. II s'agit d'une politique du cadavre que les individus acceptent largement, car la valeur de la vie est au-dessus de toute chose. Mais comme nous avons pu le mentionner, il n'existe pas de bon ton pour mentionner ce fait ni, non plus, pour parler sans tabou d'un porteur d'organe issu d'un cadavre. Un peu de respect, s'il vous plaît !

\section{Notre arrivée à la fin}

Notre voyage, littéralement parlant, nous a menés du paradis à l'enfer. D'un paradis où les morts reposent tranquillement et d'un enfer où les cadavres servent encore et toujours l'infamie des vivants. Entre les deux, suspendus dans l'ambiguïté entretenue par des vivants, nous avons également pu nous interroger sur les fantasmes, les illogismes, sinon les perversités et 
les folies, qui se poursuivent au-delà de la frontière de la mort. Quant à cette dernière, nous avons vu comment les objectifications troublées du vivant se retrouvent sous une transgression aliénante, sous une forme de «trouble » psychique. Car qui, dans une société adolescentrique, a envie de mourir?

Au-delà de nos réflexions analytiques, c'est Edgar Allen Poe qui résume le mieux tout ce qu'il y a à dire, en fin de compte, sur les cadavres (et sur les sépulcres) et le droit. Dans un poème magnifique, il nous dresse (dans la traduction de Charles Baudelaire) l'image à jamais oubliée et la leçon qui résume toute une vie, à savoir que :

Voyez ! c'est la nuit de gala.

Depuis ces dernières années désolées !

Une multitude d'anges, ailés, ornés

De voiles et noyés dans les larmes,

Est assise dans un théâtre pour voir

Un drame d'espérances et de craintes,

Pendant que l'orchestre soupire par intervalles

La musique des sphères.

Des mimes, faits à l'usage du Dieu très haut,

Marmottent et marmottent tout bas,

Et voltigent de côté et d'autre ;

Pauvres poupées qui vont et viennent

Au commandement de vastes êtres sans forme

Qui transportent la scène çà et là,

Secouant de leurs ailes de condor

L'invisible Malheur !

Ce drame bigarré ! -- oh ! à coup sûr,

II ne sera pas oublié,

Avec son Fantôme éternellement pourchassé

Par une foule qui ne peut pas le saisir,

À travers un cercle qui toujours retourne

Sur lui-même, exactement au même point !

Et beaucoup de Folie, et encore plus de Péché

Et d'horreur font l'âme de l'intrigue !

Mais voyez, à travers la cohue des mimes,

Une forme rampante fait son entrée!

Une chose rouge de sang qui vient en se tordant

De la partie solitaire de la scène !

Elle se tord ! Elle se tord !-- Avec des angoisses mortelles

Les mimes deviennent sa pâture,

Et les séraphins sanglotent en voyant les dents du ver

Mâcher des caillots de sang humain.

Toutes les lumières s'éteignent, -- toutes, -- toutes !

Et sur chaque forme frissonnante,

Le rideau, vaste drap mortuaire,

Descend avec la violence d'une tempête. 
-- Et les anges, tous pâles et blêmes,

Se levant et se dévoilant, affirment

Que ce drame est une tragédie qui s'appelle l'Homme,

Et dont le héros est le Ver conquérant. ${ }^{79}$

Il y a des choses à apprendre d’Edgar Allen Poe ! Le réalisme qui s'exprime dans ce poème est là pour nous rappeler que le Ver sera l'unique conquérant à la fin. II nous vaincra tous et pour de vrai. Si les Anges nous protègent avec ardeur dans notre vie, ils désespèrent et ne peuvent plus rien pour nous par la suite. Rien ne sera laissé, tout sera réduit à néant et nous redeviendrons poussière. Notre destin, notre avenir et notre condition humaine, c'est le Ver conquérant qui nous le rappelle. II nous rappelle que notre pèlerinage à travers la vallée des larmes, c'est la vraie nature de notre humanité. Le Ver prend, à la fin de notre pèlerinage humain, tout. Il se moque bien de notre âme, il le laisse volontiers à l'Éternel.

Tout revient, en fin de compte, au point de départ dans notre analyse, à savoir que notre attitude à l'égard du cadavre nous révèle beaucoup sur notre humanité, tant individuellement que collectivement. Elle nous illustre également le contraire, à savoir que notre narcissisme, notre aveuglement, notre nombrilisme, notre vanité, notre orgueil, notre frivolité, nous trahissent individuellement et nous déshumanisent. Nous sommes tout cela ... Et plus encore !

* Professeur, Université Laval, Québec ; bjarne.melkevik@fd.ulaval.ca.

${ }^{1}$ Quintus Septimius Florens Tertullianus, dit Tertullien (150 - 220 après J.-C.). La citation vient de « De la résurrection de la chair - chapitre IV (traduit par E.-A. de Genoude, 1852.)

${ }^{2}$ En rappelant la sagesse populaire : "Malheur à celui qui survit à ses enfants ". Le sens est bien évidemment qu'il risque de mourir seul et sans accompagnement, et par la suite être effacé de la mémoire des humains.

${ }^{3}$ Le mot « thanatologie » a historiquement eu différentes définitions. Aujourd'hui, cela fait référence à une formation technique et professionnelle pour des personnes qui se destinent à travailler dans l'industrie des pompes funèbres et c'est donc le nom d'une telle formation et une telle profession (thanatologue/ conseilleur en funéraire). Historiquement, le mot se retrouve en tant qu'observation médicale, en tant qu'analyse médicolégale ou " médecin judiciaire » (en anglais : forensic medicine), en tant que thème psychologique (et surtout psychanalytique) et psychiatrique), de même qu'un thème romantique, littéraire, poétique, etc.

${ }^{4}$ Giambattista Vico, De I'antique sagesse de I'Italie (De antiquissima Italorum sapientia ex linguae latinae originibus eruenda, Naples, 1710), Paris, Garnier-Flammarion, 1993, chapitre 1. Cf. G. Vico, La "science nouvelle" (Scienza Nuova, 1725), Paris, Gallimard, Tel, 1993. Le « verum esse ipsum factum » a été traditionnellement traduit en français comme " le vrai est le faire même ", là où nous lisons " le vrai c'est de faire le fait ". L'absence d'un " objet ", d'une réalité, de ce qu'a été fait, devient en effet préoccupant dans le constructivisme contemporain où tout devient « le faire de sujet » et donc aussi la réécriture (frauduleuse) de la réalité selon les désirs, les idéologies, les spins, favorisés par un « cercle oligarchique » ou la Ligue du politiquement et moralement correct. 
${ }^{5}$ Une pratique pourtant établie bien avant l'époque pharaonique (c.-à-d. avant la période thinite, 3150 avant J.-C). À cet avant - époque, c'était l'enterrement dans le sable qui servait le rôle de dessiccation et de conservation du cadavre.

${ }^{6}$ Hérodote, Histoire d'Hérodote (Traduction par Pierre-Henri Larcher), Charpentier, 1850 (Tome 1, livre 3, (Thalie), XVI : «En effet, les Perses croient que le feu est un dieu, et il n'est permis, ni par leurs lois, ni par celles des Égyptiens, de brûler les morts. Cela est défendu chez les Perses, parce qu'un dieu ne doit pas, selon eux, se nourrir du cadavre d'un homme : cette défense subsiste aussi chez les Égyptiens, parce qu'ils sont persuadés que le feu est un animal féroce qui dévore tout ce qu'il peut saisir, et qui, après s'en être rassasié, meurt lui-même avec ce qu'il a consumé. Or, leurs lois ne permettent pas d'abandonner aux bêtes les corps morts; et c'est par cette raison qu'ils les embaument, de crainte qu'en les mettant en terre, ils ne soient mangés des vers. "

${ }^{7}$ Giambattista Vico, «La science nouvelle " (1725) (traduction de Christina Trivulzio), Paris, Gallimard, coll. Tel, 1993, p 109. : " Remarquons d'abord que toutes les nations, barbares ou civilisés, quoique séparées par d'immenses pays, quoique fondées en des temps divers, gardent trois coutumes humaines suivantes: Toutes ont une religion; toutes contractent solennellement des mariages; toutes ensevelissent leurs morts. Chez les nations les plus sauvages et les plus cruelles, il n'est aucun acte humain qui soit célébré avec plus de pompe et de solennité que les cérémonies religieuses, les mariages et les enterrements. Nous avons dit, dans nos aphorismes, que des idées uniformes nées chez des peuples qui ne se connaissent point entre eux doivent nécessairement avoir un principe commun de vérité. Quelque chose donc a sans doute enseigné à toutes les nations ces trois commencements de l'humanité, et toutes les nations doivent observer religieusement ces coutumes, afin que le monde ne devienne pas de nouveau féroce et sauvage $"$.

À comparer avec la traduction de Jules Michelet, CEuvres choisies de Vico, Paris, 1835, p. 397 : " Observons toutes les nations barbares ou policées, quelque éloignées qu'elles soient de temps ou de lieu ; elles sont fidèles à trois coutumes humaines : toutes ont une religion quelconque, toutes contractent des mariages solennels, toutes ensevelissent leurs morts. Chez les nations les plus sauvages et les plus barbares, nul acte de la vie n'est entouré de cérémonies plus augustes, de solennités plus saintes, que ceux qui ont rapport à la religion, aux mariages, aux sépultures. Si des idées uniformes chez des peuples inconnus entre eux doivent avoir un principe commun de vérité, Dieu a sans doute enseigné aux nations que partout la civilisation avait eu cette triple base, et qu'elles devaient à ces trois institutions une fidélité religieuse, de peur que le monde ne redevînt sauvage et ne se couvrît de nouvelles forêts. " L'originale version italienne est la suivante: "Osserviamo tutte le nazioni così barbare come umane, quantunque, per immensi spazi di luoghi e tempi tra loro lontane, divisamente fondate, custodire questi tre umani costumi: che tutte hanno qualche, religione, tutte contraggono matrimoni solenni, tutte seppeliscono i loro morti ; né tra nazioni, quantunque selvagge e crude, si celebrano azioni umane con più ricercate cerimonie e più consagrate solennità che religioni, matrimoni e sepolture. Ché, per la degnità che "idee uniformi, nate tra popoli sconosciuti tra loro, debbon aver un principio comune di vero", dee essere stato dettato a tutte: che da queste tre cose incominciò appo tutte l'umanità, e per ciò si debbano santissimamente custodire da tutte perché 'I mondo non s'infierisca e si rinselvi di nuovo. Perciò abbiamo presi questi tre costumi eterni ed universali per tre primi princípi di questa Scienza ", Giambattista Vico, La scienza nuova, Milan, Einaudi, coll. La letteratura italiana Einaudi, 1976, p 129. 
Voir également Ugo Foscolo (1778 - 1827), poète italien et connu pour le poème « Dei Sepolcri » / " Les Tombeaux » de 1807 : I sepolcri ed altre poesie / Les Tombeaux et autres poèmes, trad. et présenté par Michel Orcel, Coll. Villa Médicis, Académie de France à Rome, 1982. Cf. également, Sophie Lacroix, Tombeau. Pour une archéologie de la conscience, Paris, Éditions Manucius, 2013.

${ }^{8}$ Claude Lévi-Strauss, Tristes tropiques, Paris, Plon, 1955. La chaire de recherche universitaire de LéviStrauss (1908 - 2009) à l'École pratique des hautes études (Paris) était intitulée « Chaire des religions comparées des peuples sans écriture $"$.

${ }^{9}$ Ce qui était la pratique historique des aborigènes d'Australie pendant leurs longs déplacements, leurs treks saisonniers.

${ }^{10}$ Le mot thanatopraxie désigne aujourd'hui, l'art, la science ou les techniques modernes permettant soit de préserver des corps de défunts humains de la décomposition naturelle, soit de les présenter avec l'apparence de la vie pour les funérailles et, soit, d'assurer la destruction d'un maximum d'infections et micro-organismes pathologiques contenus dans le corps des défunts. Le mot s'utilise également pour comprendre les pratiques historiques, anthropologiques et culturelles, de ces arts, sciences et techniques, concernant le traitement funéraire et/ou cérémoniel du cadavre. Voir note 3 concernant le mot thanatologie.

${ }^{11}$ Ichtyophages signifie en soi uniquement « mangeur de poissons » (ou de " fruits de mer ») et ce nom est communément attribués, en anthropologie ou en histoire anthropologique, à une pluralité de peuples se nourrissant des ressources de la mer. Sur le plan de l'histoire anthropologique le nom est surtout attribué aux peuples ancestraux au confluent de la Mer méditerranée, le Mer rouge et la Mer indienne, vivante des ressources maritimes.

12 Le mazdéisme est considéré comme le nom correct des "zoroastrismes ». En fait, le mazdéisme vénère le dieu Ahura Mazdà (" Le Seigneur Attentif »). Souvent les deux termes se juxtaposent comme profession de foi du fidèle à Ahura Mazdà, tandis que le zoroastrisme se rapporte à l'enseignement de Zoroastre (Zarathoustra) concernant cette foi. Quant à la notion de " parsis » (littéralement : " persans ») il fait référence au mazdéen (d'origine persane ou aujourd'hui iranienne) vivant en Inde (principalement en Mumbai). Le mazdéisme des parsis est souvent considéré comme un mazdéisme reformé et démystifié dans un sens éthique et monothéiste. Le mazdéisme et les mazdéens ont été cruellement discriminés et même tués en masse pendant les différents régimes islamiques en Perse/Iran. Cette situation discriminatoire perdure encore aujourd'hui : voir Vida Amirmokri, L'islam et les droits de l'homme : l'islamisme, le droit international et le modernisme islamique, Québec, Les Presses de l'Université Laval, 2004. Pour la religion mazdéenne, voir Julien Ries, La religion de Zarathustra et le mazdéisme depuis les origines jusqu'à l'avènement des Achéménides, Louvain-la-Neuve : Centre d'histoire des religions, 1983, et Julien Ries, Les religions de I'Iran sous les Achéménides et sus les Arsacides : mazdéisme, mithriacisme, zervanisme, Louvain-la-Neuve : Centre d'histoire des religions, 1984.

${ }^{13}$ Notons que le mazdéisme professe qu'il y a trois éléments sacrés - le feu, la terre et l'eau - et qu'il faut éviter toute souillure de celles-ci. L'exposition des cadavres aux oiseux répond donc au critère de ne pas souiller ces éléments, de même que de tromper (ou éloigner) le démon de la putréfaction (la « Druj Nàsu »). Soulignons cependant que les chiens remplacent historiquement les oiseaux (les vautours) dans le mazdéisme historique en Iran, Afghanistan et Pakistan/Inde.

${ }^{14}$ L'exception est bien sûr quand les oiseaux dans l'affolement laissent tomber des « restes humains » de leurs becs dans la ville. 
${ }^{15}$ Quant à la symbolique, notons le titre du livre de Graeme Smith intitulé « Et les chiens bouffent les cadavres. Notre guerre en Afghanistan " (titre original : The dogs are eating them now: our war in Afghanistan), Québec, Les Presses de l'Université Laval, 2015.

${ }^{16}$ Cela fait également référence au refus de sépulcre dans l'histoire d'Antigone et son frère Polynice, et le fait que le cadavre du dernier fut jeter aux chiens errants ce qu'Antigone n'accepta pas et qui n'était pas acceptable selon la tradition hellénique. Nous reviendrons plus loin dans notre article ici sur ce drame théâtral (et mythe) de Sophocle.

17 Bernard Vandermeersch, "Les premières sépultures ", dans Histoire et Archéologie, dossier : La Mort dans Préhistoire, no 66, septembre 1982, p 10 -14. Cf. Anne Marie Tillier, L'homme et la mort. L'émergence du geste funéraire durant la Préhistoire, Paris, Éditions CNRS, 2009 (repris, dans la coll. Biblis, 2013).

18 Bertolt Brecht, Poèmes, tome 4. (1934-1941), Paris, L’Arche éditeur, 1966. Le poème intitulé : "Questions que pose un ouvrir qui lit » (traduction par Maurice Regnaut) :

«Oui a construit Thèbes aux sept portes?

Dans les livres, on donne les noms des Rois.

Les Rois ont-ils traînés les blocs de pierre ?

Babylone, plusieurs fois détruite,

Qui tant de fois l'a reconstruite ? Dans quelles maisons

de Lima la dorée logèrent les ouvriers du bâtiment ?

Quand la Muraille de Chine fut terminée,

Où allèrent, ce soir-là, les maçons ? Rome la grande

Est pleine d'arcs de triomphe. Qui les érigea ? De qui

Les Césars ont-ils triomphé ? Byzance la tant chantée,

N'avait-elle que des palais

Pour ses habitants ? Même en légendaire Atlantide,

Hurlant dans cette nuit où la mer l'engloutit,

Ceux qui se noyaient voulaient leurs esclaves.

[..............

Autant de récits,

Autant de questions. »

19 Peut également s'écrire comme Qin Shi Huang, Qin Shi Huangdi ou Roi/Empereur Zheng de Qin. Rappelons que son nom de famille était Ying, son prénom Zheng, son nom de clan Qin (ou Zhao). Soulignons que le nom Shi Huang signifie, littéralement, le Premier August Souverain (d'où s'ajoute par devant le lieu Qin). L'utilisation du titre Shi Huangdi fait référence aux souverains légendaires de I'antiquité chinoise, les Sanhuangwudi.

L'Empereur de Qin a eu une influence énorme pour la philosophie du droit en Chine comme prometteur du légisme chinois (Fa Jia); sur cette question, voir Bjarne Melkevik, " Un regard sur la culture juridique chinoise: l'École des légistes, le confucianisme et la philosophie du droit », dans idem, Horizons de la philosophie du droit, Paris, L'Harmattan et Québec, Les Presses de l'Université Laval, 1998 p 179-203 (repris dans, idem, Philosophie du droit. Volume 1, en 2010) ; idem, « À propos de la philosophie chinoise du droit ", dans, idem, Considérations juridico-philosophiques, Québec, Les Presses de l'Université Laval, 2005, p 171-178 (repris dans, idem, Philosophie du droit. Volume 2, Québec, Les Presses de l'Université Laval, 2014) ; traduction de l'article initial en langue anglaise "Chinese Legal Philosophy ", dans Christopher Barry Gray (dir.), The Philosophy of Law : An Encyclopedia, volume 1, New York, Garland Publishing, 1999, p 104-107. 
${ }^{20} \mathrm{Cf}$. Renata Pisu, L'armée ensevelie de l'empereur Qin : la plus grande découverte archéologique (traduction et adaptation de l'italien par Christian Pessey de "I soldati dell'imperatore Qin Shi Huang "), Paris, Solar, 1985 ; Arthur Cottorell, Le premier empereur : la plus grande découverte archéologique du siècle, Paris, Chêne/Hachette, 1981.

${ }^{21}$ Nous utilisons ici le terme « hollywoodien » dans un sens générique (et emblématique) vu que plusieurs de ces séries télévisuelles ne sont pas strictement parlant produit à Hollywood. En vérité, la production à la façon " hollywoodienne » est aujourd'hui devenue un phénomène mondial, prenant plusieurs formes, dont celle d'imitation et d'acculturation. Les productions " hollywoodiennes " se font à présent autant en Inde qu'en Australie, ou encore en France, Angleterre, Italie, Allemagne, Égypte, Nigeria, Chine, Brésil, Canada, etc., et à l'égal de ce qui se produit véritablement à Hollywood.

22 La série de télévision "Six Feet Under » a été produite par Alan Ball pour HBO. De 2001 jusqu'à l'automne 2005, la série a connu 5 saisons et 63 épisodes. Le titre "Six pieds sous terre " a été utilisé dans le monde francophone.

${ }^{23}$ Notons qu'historiquement et anthropologiquement les " six pieds sous terre " étaient la mesure de protection contre les animaux charognards qui pouvait déterrer un cadavre. Les charognards n'étaient pas capables d'aller jusqu'à « six pieds sous terre » pour festoyer sur le cadavre.

${ }^{24}$ La série de télévision "Dead Like Me " (Mort comme moi) a été produite par Bryan Fuller pour Showtime. De 2003 jusqu'à 2004, la série a connu deux saisons et 29 épisodes.

${ }^{25}$ La télésérie " Bones » est inspiré des romans de Kathleen Joan Toelle " Kathy » Reichs.

${ }^{26}$ Le titre "Six pieds sous terre » reprend l'obligation ancienne de déposer le cadavre si profond que les animaux charognard ne pouvait pas le déterrer. La phase " enterrer six pieds sous terre " a bien le sens d'un cadavre " enterrer correctement ».

${ }^{27}$ Notons que les écrits du poète, écrivain et croquemort Thomas Lynch, directeur des pompes funèbres de Lynch \& Sons funeral home de Milford, Michigan, est la source d'inspiration de la série. Thomas Lynch est l'auteur de The Undertaking : Life Studies from a Dismal Trade (Penguin, 1998) et Bodies in Motion and at Rest (Norton, 2001), de même que trois livres de poésie qui tourne également autour des cadavres, des pompes funèbres et la vie dans toutes ses imperfections. Les livres de Lynch respirent de I'honnêteté, des aléas de l'existence et un sens aigu d'humour quand il traite de lui-même (son alcoolisme, son divorce, ses origines irlandaises, sa formation de catholique irlandais, etc.) et son travail de croquemort " en poésie ", autrement dit en tant qu'auteur poétique croquemort.

${ }^{28}$ D'où le néologisme de " adulescent » (" adolescence »), conçu par la fusion des mots " adulte » et " adolescent ». Autrement dit le paradigme de "Peter Pan ».

${ }^{29}$ Robert Musil, L’homme sans qualité, 2 tomes, Paris, Seuil, 1954.

30 Rappelant le poème de John Dunn (1572 -1631, Devotions upon Emergent Occasions, 1624 (Méditation XVII - Nunc lento sonitu dicunt, Morieris; - Now this bell tolling softly for another, says to me, Thou must die):

" Aucun homme n'est une île, un tout, complet en soi ;

tout homme est un fragment du continent,

une partie de l'ensemble ;

si la mer emporte une motte de terre,

l'Europe en est amoindrie,

comme si les flots avaient emporté un promontoire,

le manoir de tes amis ou le tien ; 
la mort de tout homme me diminue, parce que j'appartiens au genre humain ; aussi n'envoie jamais demander pour qui sonne le glas :

c'est pour toi qu'il sonne. »

Les mots de la fin (et le sous-titre "Nunc lento sonitu dicunt, Morieris » [Maintenant, cette cloche sonne doucement pour une autre; elle me dit: Tu dois mourir], de ce poème a inspiré Ernest (Miller) Hemingway (1899-1961) pour le titre de son roman : For Whom the Bell Tolls (« Pour qui sonne le glas ») 1940.

${ }^{31}$ Sed contra, Bjarne Melkevik, Épistémologie juridique et déjà-droit, Paris, Buenos Books International, 2014.

${ }^{32}$ La tradition historique de I'hindouisme interdite la mise en terre du cadavre. II existe pourtant des dissidents surtout géographiques et « des sectes » qui acceptent une telle pratique.

33 Sophocle, Théâtre complet, traduction de Robert Pignarre, Paris, GF Flammarion no 18, 1964, pages 69 - 101 (Antigone). Cf. Muriel Gilbert (dir.), Antigone et le devoir de sépulture, Genève, Labor et Fides, 2015 et surtout l'article de Pierre Moor, "Entre Antigone et Créon : la médiation juridique », idem, page $99-118$.

${ }^{34}$ Voir, Stamatios Tzitzis, "Scolies sur les nomima d'Antigone, représentés comme droit naturel », dans Archives de philosophie du droit, tome 33, 1988, p 243-259. Fabio Ciaramelli, " Pour une relecture de l'Antigone comme tragédie du nomos ", dans Metodo. International Studies in Phenomenology and Philosophy, Vol. 2, n. 1 (2014), page 201 - 215. Voir aussi Paulo Ferreira da Cunha, O século de Antigona, Coimbra, Almedina, 2003.

${ }^{35}$ Sophocle, Théâtre complet, op. cit., page 70.

${ }^{36}$ Le fait qu'Antigone jette le sable sur le corps de son frère mort pour symboliser un sépulcre, rappelle l'explication de Cicéron des coutumes sacrées romaines dans De Legibus (Traité des lois) : « Car avant que la terre n'ait été répandue sur un ossement du défunt, le lieu sur lequel a été brûlé son corps ne comporte pas d'égards religieux; mais quand la terre a été répandue, le défunt est alors inhumé en cet endroit : le lieu s'appelle « Tombeau » (sepulcrum) et désormais un grand nombre de droits religieux le concernent. ", Cicéron, Traité des lois, (traduction de Georges De Plinval), Paris, Les Belles Lettres, 1968, Livre deuxième, XXII - 57. Notons que la même tradition existe symboliquement aujourd'hui, dans le fait qu'un prêtre chrétien ou le plus prochain parent prend un poigné de " terre » et le répande sur le lieu d'enterrement. Par cet acte, il s'agit aujourd'hui comme il y a 2.400 ans de désigner un « tombeau », d'un « sépulcre ».

37 Une libation est acte rituel religieux qui nous vient de l'Antiquité. Elle s'effectue par la présentation d'une boisson en offrande (à un Dieu) et renversante quelques gouttes sur le sol ou sur l'autel. Les liquides de libation étaient le plus souvent soit du vin, du lait ou de I’huile d'olive. La Sainte Bible témoigne également de cette pratique : 2 Timothée 4 : «Mais toi, sois sobre en toutes choses, supporte les souffrances, fais l'œuvre d'un évangéliste, remplis bien ton ministère. Car pour moi, je sers déjà de libation, et le moment de mon départ approche. J'ai combattu le bon combat, j'ai achevé la course, j'ai gardé la foi... ".

38 Sophocle, Théâtre complet, Antigone, op. cit., page 79.

${ }^{39}$ Sophocle, op. cit., p. 70.

40 L'institution de sati est aujourd'hui interdite par la loi en Inde. L'histoire de cette interdiction législative remonte à 1829 et Regulation XVII (Sati Prevention Regulation Act) de Lord William 
Bentinck qui la fait incriminer. C'est le résultat d'une campagne contre la politique orientaliste de l'époque et le dogme de respecter sans jugement toutes les coutumes ancestrales et religieuses de I'Inde. Cf. Catherine Weinberger-Thomas, Cendres d'immortalité : La crémation des veuves en Inde, Paris, Seuil, 1996. L'institution de sati (auto-immolation des veuves) se comprend avec une référence à l'institution du jauhâr (ou johur), la coutume de mort volontaire sur le bûcher funéraire suivie par les femmes des guerriers râjputs (et d'autres peuples également) afin d'éviter la déportation, la soumission et l'esclavage pendant les conquêtes musulmanes en Inde.

${ }^{41}$ Cicéron (Marcus Tullius Cicero - 106 av. J. C - 43 av J. C.), Traité des lois, (traduction de Georges De Plinval), Paris, Les Belles Lettres, 1968, Livre deuxième, XXII - 56. Remarque également la conclusion de Cicéron sur cet acte : " C'est peut-être de peur que la même insulte n'arrivât à son corps qu'il fut le premier des patriciens de la gens Cornelia à vouloir qu'on le brûlât par la flamme " (57).

${ }^{42}$ Voir sur les génocides, les massacres, les tueries en masse, des Arméniens, voir : Annette Becker, Hamit Bozarslan et Vincent Duclert, Le génocide des Arméniens - Un siècle de recherche 1915-2015, Paris, Armand Colin, coll. "Hors collection ", 2015. Si les études contemporaines ciblent les génocides des Arméniens de 1914/5 à 1921, il convient de noter que les tueries en masse des Arméniens commencent dans le 19e siècle. II s'agit d'effacer la présence arménienne de l'Empire ottoman et de la Turquie. La discrimination contre la minorité Arméniens en Turquie est aujourd'hui encore systématique et endémique.

${ }^{43}$ Voir la collection de livres: "Les cadavres dans les génocides et les violences de masse ", Paris, Éditons Pétra. Elisabeth Anstett et Jean-Marc Dreyfus, Cadavres impensables, cadavres impensés. Approches méthodologiques du traitement des corps dans les violences de masse et les génocides, Paris, Éditons Pétra, 2012; Anne-Marie Losonczy et Valérie Robin Azevedo, Retour des corps, parcours des âmes. Exhumations et deuils collectifs dans le monde hispanophone, Paris, Éditons Pétra, 2016. Cf. aussi Sévane Garibian (dir.), La mort du bourreau: Réflexions interdisciplinaires sur le cadavre des criminels de masse, Paris, Éditons Pétra, 2016.

${ }^{44}$ Et d'où aussi le phénomène historique consistant d'utiliser un cadavre en tant que pharmacopée. Ce qu'était coutumière dans l'Égypte pharaonique à l'Égypte islamisée où les corps momifiés étaient profanés et vendus, en petit morceau, en tant que " médicament ", comme remède médical, comme pharmacopée, à des gens qui croyaient, contre raison, que cela leur permettait de guérir.

${ }^{45} \mathrm{Cf}$. Mark Twain, Robert Louis Stevenson, etc.

${ }^{46} \mathrm{Cf}$. le statut de "l'enfant sans vie » en droit civil français, Code civil (française) l'article 79-1 (voir surtout alinéa 2) : "Lorsqu'un enfant est décédé avant que sa naissance ait été déclarée à l'état civil, l'officier de l'état civil établit un acte de naissance et un acte de décès sur production d'un certificat médical indiquant que l'enfant est né vivant et viable et précisant les jours et heures de sa naissance et de son décès.

À défaut du certificat médical prévu à l'alinéa précédent, l'officier de l'état civil établit un acte d'enfant sans vie. Cet acte est inscrit à sa date sur les registres de décès et il énonce les jour, heure et lieu de l'accouchement, les prénoms et noms, dates et lieux de naissance, professions et domiciles des père et mère et, s'il y a lieu, ceux du déclarant. L'acte dressé ne préjuge pas de savoir si l'enfant a vécu ou non ; tout intéressé pourra saisir le tribunal de grande instance à l'effet de statuer sur la question. » Alinéa 2 ici permet d'obtenir un "acte d'enfant sans vie " ou "Certificat médical d'accouchement : en vue d'une demande d'établissement d'un acte d'enfant sans vie ». 
${ }^{47}$ Concernant le mode et l'appréciation contemporaine de transgressions, voir Jean-Jacques Sueur et Pascal Richard (dir.), La transgression, Bruxelles, Bruylant, 2013, et surtout notre article " Le truisme du droit comme transgression », ibid., p $453-478$.

${ }^{48}$ Et de philosophie, surtout présente dans la philosophie des Lumières où la figure du gentil cannibale (en exemple du Bon Sauvage des Philosophes) est opposée à l'homme occidental barbare, cruel et non civilisé (ce qui est aujourd'hui repris à l'identique par " le progressisme réactionnaire " en tant que mythologie du l'homme occidental WASP ou encore raciste, antiféministe, et pire). Le mythe du gentil cannibale se trouve, par exemple, dans les écrits de Girolamo Cardano (1501 -1576) et Michel de Montaigne (1533 - 1592); voir sur cette question Frank Lestringant, Le Cannibal. Grandeur et décadence, Genève, Droz, 2016. L'essai de Michel de Montagne, Des Cannibales, Paris, Mille et une nuits, 2000, est un extrait (livre 1, chapitre 31) de son livre « Essais » (1580).

${ }^{49}$ Khalid Ghanayim, "Excused Necessity in Western Legal Philosophy», dans The Canadian Journal of Law and Jurisprudence. An International Journal of Legal Thought, London (Ontario), University of Western Ontario, 2006, vol. 19, no 1, p $31-65$.

${ }^{50} \mathrm{Cf}$. sur le plan littéraire (sans suite judiciaire), Henrik Ibsen, "Peer Gynt", Paris, Flammarion, coll. GFFlammarion no 805, 1994, p 243-246 (Acte V). C'est la scène d'un naufrage, d'une tempête et deux personnes qui s'accroche tant bien que mal à une quille. Peer Gynt après avoir précipité l'autre naufragé vers la noyade, se hisse seul sur la quille en déclamant « Amen, mon gars. Tu étais et restas toi-même jusqu'à la fin. Tant qu'il y a de la vie, il y a de l'espoir. " (Idem, page 246).

${ }^{51}$ R. v. Dudley et Stephens (1884, 14 QBD 273 DC). Voir, Alfred William Brian Simpson, Cannibalism and the Common Law: The Story of the Tragic Last Voyage of the Mignonette and the Strange Legal Proceedings to Which It Gave Rise, Chicago: University of Chicago Press, 1984. Cf. Alfred William Brian Simpson, "Cannibals at Common Law", The University of Chicago Law School, Crosskey Lectures, No. 5 (1981).

52 C'est-à-dire que le privilège d'être sauvé (de se sauver avec les moyens disponibles) ne pouvait pas être discriminé au préalable à partir d'un a priori. D'où précisément un positionnement relevant de l'égalité.

${ }^{53}$ Ce qui effectivement a été plus « juste ». Voir Edgar Allan Poe, Les aventures d'Arthur Gordon Pym de Nantucket (original 1838, trad. fr. 1858), chapitre XII (" La courte paille ».) : " Je revins à temps de mon évanouissement pour voir le dénouement de la tragédie et assister à la mort de celui qui, comme auteur de la proposition, était, pour ainsi dire, son propre meurtrier. Il ne fit aucune résistance, et, frappé dans le dos par Peters, il tomba mort sur le coup. Je n'insisterai pas sur le terrible festin qui s'ensuivit immédiatement : ces choses-là, on peut se les figurer, mais les mots n'ont pas une vertu suffisante pour frapper l'esprit de la parfaite horreur de la réalité. Qu'il me suffise de dire qu'après avoir, jusqu'à un certain point, apaisé dans le sang de la victime la soif enragée qui nous dévorait, et détaché d'un commun accord les mains, les pieds et la tête, que nous jetâmes à la mer avec les entrailles, nous dévorâmes le reste du corps, morceau par morceau, durant les quatre jours à jamais mémorables qui suivirent, 17, 18, 19 et 20 juillet. » Cf. Edgar Allan Poe, Histoires, Essais et Poèmes, Paris, Le livre de poche, La Pochothèque, 2006, p $1231-1473$, page 1372 pour la citation.

${ }^{54}$ Quant à la question morale de ce cas nous pouvons nous rapporter à Edmond Cahn, The Moral Decision, Bloomington, Indiana University Press, 1956, p 61 s. Voir également, idem, The Sense of Injustice, New York \& London, 1949, et, idem (dir.), Supreme Court and supreme law, Bloomington, Indiana University Press, 1954. 
55 II s'agit du Jeffrey Dahmer. Voir, Robert J. Dvorchak et Lisa Holewa, Milwaukee Massacre: Jeffrey Dahmer and the Milwaukee Murders, London, Robert Hale, 1992

56 II s'agit d'Armin Meiwes. Voir, Rainer Maria Kiesow, L'Unité du droit, Paris, Éditions EHESS, 2014, p $73-75$.

${ }^{57}$ Plusieurs films ont été produits sur l'affaire de Jeffrey Dahmer (en 1993 et en 2002), de même que des références à son nom et ses crimes ont été faits dans plusieurs séries de télévision. Jeffrey Dahmer est incontestablement aujourd'hui un " must ", une " icône ", une figure de " culte " révéré dans le contre-culturel " underground ». Une banalisation « culturelle » glorifiante est actuellement en œuvre; par exemple, dans la bande dessinée de John "Derf" Backderf, intitulé "mon ami Dahmer » (2013), et également dans le film basé sur cette bande dessinée et qui est sorti en salle en avril 2017.

58 Jugement de la Cour fédérale / Bundesgerichtshofes du 22 avril 2005, ref. Az: 2 StR 310/0. Un deuxième procès à la Cour d'assises a eu lieu et le 9 mai 2006, Armin Meiwes a été condamné à la réclusion à perpétuité pour assassinat et pour la perturbation de la paix des morts. La Cour fédérale a confirmé cette décision en février 2007. Un recours pour atteinte aux "droits constitutionnels » n'a pas été accepté - par ordonnance du 7 octobre 2008 - par la Cour constitutionnelle fédérale d'Allemagne.

${ }^{59}$ Cf. American Psychiatric Association, DSM-5: diagnostic and statistical manual of mental disorders, 5e édition, Washington D.C. American Psychiatric Association, 2013. La quatrième édition a été traduite en langue française : DSM-IV-TR, Manuel diagnostique et statistique des troubles mentaux, texte révisé, Paris, Elsevier Masson, 2004 (1002 pages). On retrouve encore mieux avec le livre classique de Richard von Krafft-Ebing, Études médico-légales : Psychopathia Sexualis. Avec recherche spéciale sur l'inversion sexuelle, traduite à partir de la 8e édition allemande par Émile Laurent et Sigismond Csapo, Paris, Éd. Georges Carré, 1895 (librement disponible sur la Toile: le projet Gutenberg).

${ }^{60}$ Sigmund Freud, Éros et Thanatos ; cf. également, idem, Pulsions et destin des pulsions (1915), Paris, Payot, coll. Petite Bibliothèque Payot, 2010

${ }^{61}$ Richard von Krafft-Ebing, Études médico-légales : Psychopathia Sexualis. Avec recherche spéciales sur I'inversion sexuelle, traduite sur la 8e édition allemande par Émile Laurent et Sigismond Csapo, Paris, Éd. Georges Carré, 1895 (librement disponible sur la Toile : le projet Gutenberg), article " Outrages aux cadavres (Nécrophiles) ".

62 Lynne Stopkewich (réalisatrice) "Kissed » (le titre québécois était "Extase »), Montréal 1996. Les acteurs principaux étaient Molly Parker, Peter Outerbridge, Jay Brazeau, James Timmons, Jessie Winter Mudie, Annabel Kershaw. Le film, l'adaptation d'une nouvelle de Barbara Gowdy "We So Seldom Look on Love" (1992), fût présenté à Cannes dans Quinzaine de réalisateurs du Festival de Cannes en 1997. Le film est sorti en DVD en 2005 et en 2007. Le film a été programmé à plusieurs reprises dans les stations de télévision au Canada et ailleurs.

63 Le grand nom de mouvement immortaliste est Robert Chester Wilson Ettinger (1918 - 2011), feu professeur de physique américain. II publia ces thèses dans son livre, L'homme est-il immortel ?, Paris, Denoël, 1964 (traduction française de The Prospect of Immortality, 1962, 1964, etc.) Le professeur Ettinger est un partisan prônant que la science peut servir, dans une perspective de l'immortalisation à réanimer le corps mort, à un moment opportun dans le futur, pour éventuellement à ce moment-là, le guérir de la maladie qui l'a tuée ou encore pour réparer dans le 
futur la vieillesse avec des méthodes plus appropriées que nous fournis la science de l'avenir. Il est normalement considéré comme fondateur d'une crypto secte néo-païenne (dite : les immortalistes) prônant l'immortalité de l'homme par le biais de la "Science ", sinon aussi de "transhumanisme " (c.-à-d. amélioration de l'homme par l'ingénierie génétique) en vue de devenir " parfait »! Sur ce dernier point voir, R. C. W. Ettinger, Man Into Superman: The Startling Potential of Human Evolution - And How to Be Part of It, Oak Park (MI), Immortalist Society, 1989 (2005). Comme il se doit (sic!), son corps mort fut « cryoconservé ».

${ }^{64}$ Bernard Edelman, Essai sur la vie assassinée. Petite histoire de l'immortalité, Paris, Hermann, 2016.

${ }^{65}$ Cryogénisation est libre (et légale) aux États-Unis. La France par contre vient de l'interdire par des décisions judiciaires: Voir, l'arrêt de la Cour administrative d'appel de Bordeaux du 29 mai 2000 (CAA Bordeaux 29 mai 2000, req. n 99BX02454; confirmant lui-même un jugement du tribunal administratif de Saint-Denis de la Réunion rendu le 21 octobre 1999 : TA Saint-Denis, 21 oct. 1999 : JCP G 2000, II, 10287, note F. Lemaire), le Conseil d'État a décidé dans un arrêt « Leroy » du 29 juillet 2002 (req. $n^{\circ}$ 222180). Dans le même sens, le Tribunal administratif de Nantes a rendu un jugement (TA Nantes, 5 septembre 2002, req. $n^{\circ}$ 0201396) interdisant la cryogénisation du docteur Martinot et imposant l'inhumation de ce dernier ainsi que sa femme (déposée dans un congélateur dans la crypte du château familial depuis 1984), confirmant ainsi la décision du juge des référés du Tribunal de grande instance de Saumur du 13 mars 2002. Il s'agit ici d'une précision judiciaire des dispositions de la réglementation française des opérations funéraires qui interdit ce mode de conservation du corps, puisque le cercueil contenant le corps du défunt doit être inhumé ou incinéré dans les 6 jours suivants le décès (art. R. 2213-33 et R. 2213-35 du Code Général des Collectivités Territoriales).

${ }^{66} C^{\prime}$ est la procédure de la cryogénisation du corps entier que nous venons de donner. II n'y a pas pour autant de restriction voulant que ce vous pouvez faire en gros ne peut pas également aussi être fait en détail. Les partisans de cryogénisation font donc aussi campagne pour cryogéniser des têtes, des poumons, des cœurs, et tout ce que vous souhaitez garder pour l'éternité.

${ }^{67}$ De nombreux adeptes (c.-à-d. cadavres) de cryogénisation " attendent " déjà dans des capsules climatisées. Le plus connu est Walt Disney (le fondateur des Studios Disney et les produits culturels "Disney »).

${ }^{68}$ C'est le mot qu'il, Gunther Von Hagens, utilise pour caractériser sa technique, sa " méthode de production ".

${ }^{69}$ Nous aurons aussi pu mentionner l'artiste chinois Zhu Yu (et le groupe d'art "Cadavre »). Zhu Yu utilise des cadavres " achetés » des prisons chinoises pour faire ses sculptures.

70 Le corps momifié - l'auto-icône - de Jeremy Bentham est conservé (en apparence) dans la bibliothèque universitaire de l'University College de Londres. La tête a cependant été enlevée et remplacée par une maquette, les parties musculaires et organes « moues » ont été remplacés par du " papier ", et tout est mis en scène par une structure métallique. C'est aujourd'hui une attraction touristique (et de voyeurisme). Mentions ici également le Mausolée Lénine à Moscou.

${ }^{71}$ Marc Jimenez, La querelle de l'art contemporain, Paris, Gallimard, coll. Folio Essais, 2005, p 291, voir aussi les pages $48-49$ (intitulé : Les cadavres exquis de Günther Von Hagens).

72 Aldous Huxley, Le meilleur des mondes, Paris, Pocket, coll. 1438, 2005.

${ }^{73}$ Le Monde, daté du 6 août 1993. Il y a plusieurs exemples plus récents sur cette technique; entre autres au Québec en août 2016.

${ }^{74}$ Le Monde, daté du 6 août 1993 ; la signature J.-Y. N. s'appose à cette vignette. 
75 Jean-Noël Fabiani, Ces histoires insolites qui ont fait la médecine : Tome 2 : Les transplantations, Paris, Plon, 2012.

76 Le trafic d'organes ne se fait pas seulement avec des cadavres. Pensons à ce qu'il se passe en Amérique du Sud où des gens disparaissent quelques jours pour réapparaître, mal en point, avec de l'argent dans leurs poches et quelques « organes » de moins.

77 Gary Becker a reçu le Prix Nobel de l'économie (officiellement : Nobel Memorial Prize in Economic Sciences) en 1992.

78 Gary Becker et Julio Elias, "Introducing Incentives in the market for Live and Cadaveric Organ Donations", Journal of Economic Perspectives. American Economic Association. 21. (3) (Summer 2007): 3-24. Doi:10.1257/jep.21.3.3. JSTOR 30033732.

${ }^{79}$ Edgar Allan Poe, Le ver conquérant, traduit par Charles Baudelaire (poème aussi connu sous le titre "Le ver vainqueur »). L'original s'intitule "The Conquerant Worm » fut publié dans Graham's Lady and Gentleman's Magazine en janvier 1843, et plus tard reprise dans la nouvelle "Ligeia " (2e version de 1845 ; qui est la source de la traduction de Charles Baudelaire) et par des collections variées de poésie d'Edgar Allan Poe. Cf. Edgar Poe, Histoires Extraordinaires, Paris, Michel Lévy, 1856; repris par Gallimard, Paris, collection Folio classique no 4081, (1973) 2004, p 330 - 331; ou Edgar Allen Poe, Tous les contes d'Edgar Allen Poe, Histoires Extraordinaires, Nouvelles Histoires Extraordinaires, Histoires Grotesques et sérieuses, Bruxelles, Bibliothèque Marabout no 109, 1960, p 220. 